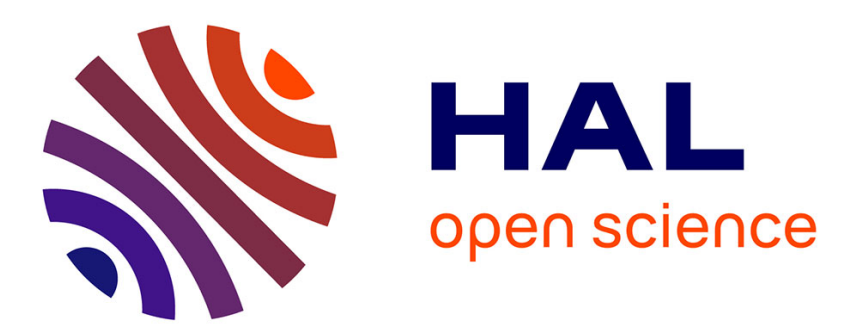

\title{
Variational Contrast Enhancement of Gray-Scale and RGB Images
}

\author{
Fabien Pierre, Jean-François Aujol, Aurélie Bugeau, Gabriele Steidl, \\ Vinh-Thong Ta
}

\section{- To cite this version:}

Fabien Pierre, Jean-François Aujol, Aurélie Bugeau, Gabriele Steidl, Vinh-Thong Ta. Variational Contrast Enhancement of Gray-Scale and RGB Images. Journal of Mathematical Imaging and Vision, 2016. hal-01237038v4

\section{HAL Id: hal-01237038 \\ https://hal.science/hal-01237038v4}

Submitted on 14 Jan 2016

HAL is a multi-disciplinary open access archive for the deposit and dissemination of scientific research documents, whether they are published or not. The documents may come from teaching and research institutions in France or abroad, or from public or private research centers.
L'archive ouverte pluridisciplinaire $\mathbf{H A L}$, est destinée au dépôt et à la diffusion de documents scientifiques de niveau recherche, publiés ou non, émanant des établissements d'enseignement et de recherche français ou étrangers, des laboratoires publics ou privés. 


\title{
Variational Contrast Enhancement of RGB Images *
}

\author{
Fabien Pierre Jean-François Aujol Aurélie Bugeau \\ Gabriele Steidl Vinh-Thong Ta
}

November 30th 2015

\begin{abstract}
The aim of this paper is two-fold. First, we propose a new method for enhancing the contrast of gray-value images. We use the difference of the average local contrast measures between the original and the enhanced images within a variational framework. This enables the user to control the contrast level and the scale of the enhanced details intuitively. Moreover, our model avoids large modifications of the original image histogram. Thereby it preserves the global illumination of the scene and can cope with large areas having similar gray values. The minimizer of the proposed functional is computed by a gradient descent algorithm in connection with a polynomial approximation of the average local contrast measure. The polynomial approximation is done via Bernstein polynomials and leads to to a speed up of the algorithm by applying fast Fourier transforms.

In the second part, the approach is extended to a variational enhancement method for color images. The model approximately preserves the hue of the original image and includes additionally a total variation term to correct possible noise. The method requires no post- or pre-processing. The minimization problem is solved with a hybrid primal-dual algorithm.

Numerical experiments demonstrate the efficiency and the flexibility of the proposed approaches in comparison with state-of-the-art methods.
\end{abstract}

\section{Introduction}

Contrast enhancement of color images that are over-lighted or under-lighted is an active field of research. The task itself is old and has been historically treated manually in a darkroom from the film negative. This technique was called dodge and burn. The dodging decreases the exposure for areas of film negatives that the photographer wants lighter, while the burning increases the exposure in order to make it darker. Digital image editing reproduces the same technique in a computational way. Nevertheless, it usually requires manual intervention by a user, which can be tedious since most parts of the image have to be edited.

\footnotetext{
*This study has been carried out with financial support from the French State, managed by the French National Research Agency (ANR) in the frame of the Investments for the future Programme IdEx Bordeaux (ANR-10-IDEX-03-02). J-F. Aujol is a member of Institut Universitaire de France. Many thanks to M. Bertalmio for providing the code of [7].
} 
In recent years, many contrast enhancement techniques have been proposed for digital images. Some approaches exploit multiple views or sensors and are able to improve image contrast in low light conditions $[43,53]$. Other methods, called sharpening, focus on enforcing the strong contours in order to remove blur obtained, e.g., by Gaussian convolution [39]. This kind of enhancement concerns only the strong image contours while contrast enhancement attempts to modify the gray level of the objects not only in the neighborhood of the contours.

In this paper, we focus on the problem of contrast enhancement of natural images captured with a digital camera. Existing methods can be divided into three groups: spectral methods, histogram methods, and spatial methods.

Spectral methods. These methods rely either on wavelets or homomorphic filtering. Laine et al. [30] proposed a method based on the wavelet transform, performing the image enhancement in the wavelet domain with a non-linear operator applied to the wavelet coefficients. This operator performs both denoising and enhancement. The denoising is achieved by soft and/or hard thresholding of high frequency bands. The other bands are modified by a piecewise affine mapping extending the expression of soft thresholding.

In [24], homomorphic filtering is performed after a logarithmic transformation. Basically, the homomorphic filter is a high-pass filter which enforces the sharpness of the image. The method can produce some Gibbs-like artifacts or a noise amplification. Moreover, the tuning of the scale and the level of the contrast enhancement is not intuitive.

Histogram methods. Histogram Specification (HS) transforms the grayscale input image to an output having a previously specified histogram. A special case of HS is Histogram Equalization, where the specified histogram is the uniform one. These methods have been well studied in the literature. The method of Mignotte [34] performs HS based on the distribution of the first order derivative in order to enforce edges. In [51], the authors propose a Partial Differential Equation (PDE) to modify the histogram. This approach tackles with the issue of specifying a particular histogram. The PDE scheme performs both denoising and contrast enhancement. Sun et al. [54] suggest a HS technique which takes the first and the second order derivatives of the image into account to preserve the shapes in the original image.

Some methods use a prior on the histogram. Wang et al. [56] address a prior that maximizes the entropy of the output image. Arici et al. [3] propose a variational framework which makes a trade-off between the histogram of the input image and the uniform one. A smoothing term is added to penalize discontinuities of the histogram.

In order to avoid problems in multi-modal histograms, some approaches divide the histogram specification into sub-histogram specifications. In [29], the values of the original image are split in order to equalize two sub-histograms. The splitting is achieved on both sides of the mean. Chen et al. [16] propose to perform this separation recursively. The algorithm of Sim et al. [52] uses the same division but based on the median. In [1], Wadud et al. divide the histogram into multiple sub-histograms in order to equalize separately the complete histogram while protecting small features of the input image. With the same 
idea, Celik et al. [12] model the histogram as a mixture of Gaussians. For more division strategies we refer to [28] and the references therein. Some methods are adaptive because the enhancement is done locally, see, e.g., [10, 11, 25, 33]. However, in these methods the influence of neighboring and far away pixels on the enhanced one is the same.

A problem of histogram techniques is the meaningful strict ordering of the pixel values. If such ordering is available, the HS can be done in a straightforward way, see, e.g., [15]. Several methods to obtain a meaningful ordering were developed as the local mean ordering [17], the wavelet based ordering [55], and the variational approach based on the minimization of a fully smoothed $\ell_{1}$-TV functional $[15,38]$ together with its fast version [37].

Finally, we mention that HS can also be done on the color histogram, for instance by the variational framework [41] which requires a target color image with desired contrast. A two step algorithm consisting of a HS of the intensity image, followed by a hue and range preserving color adjustment was proposed by Nikolova et al. in [35, 36]. For a toolbox see [26].

Spatial methods. These approaches tackle with the problem of most histogram approaches which change the values of pixels without considering spatial constraints. A first approach of Boccignone [8] uses the anisotropic diffusion equation of Perona and Malik [42] in a multi-scale framework.

Most spatial methods take hypotheses about the Human Visual System (HVS) into account. In particular, the perceptual work about the Retinex model [31] has found wide acceptance. Two remote pixels with the same value can be perceived by a human as having different intensities. For example, Figure 1 shows the experiments of the Checker shadow illusion of Adelson [2]. Although the squares A and B are physically of the same intensity, the perceived intensities are different. Because of this difference in perception, a contrast enhancement could be performed by changing their values, darker for the darkest perceived A, and lighter for the lightest perceived B. The perceived intensity would be the same, but the local contrasts would be improved.

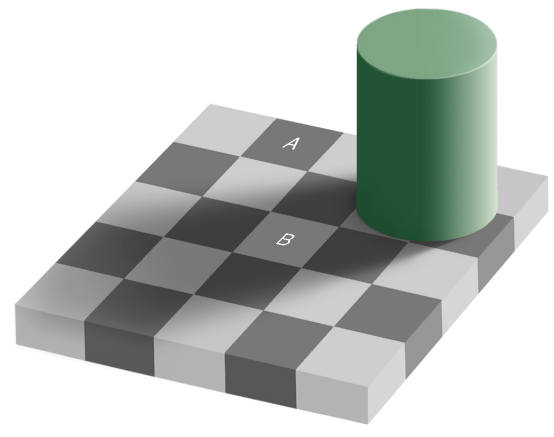

(a) Checker shadow illusion of Adelson

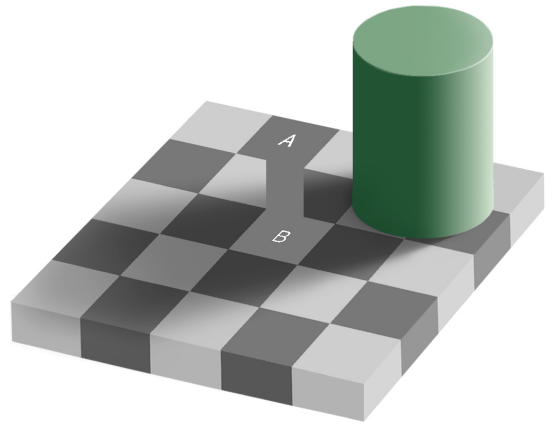

(b) Proof of illusion

Figure 1: Checker shadow illusion of Adelson [2]. The two squares A and B are of the same value, but their gray levels are perceived as different by the Human Visual System. This failure is taken as an advantage by the method proposed in this paper to enforce the contrasts of images. 
Rizzi et al. [48] propose an approach, called Automatic Color Equalization (ACE), based on a perceptual hypothesis. Bertalmio et al. [7] integrate the previous approach into a variational model. The method was generalized by Palma-Amestoy et al. [40] for a larger class of functionals. This last method was implemented in [18]. Provenzi et al. [47] propose to work in the wavelet domain in order to reduce the computation time. Some links between these works and the Retinex model have been studied in [6]. Since these approaches are related to our new model, we explain them more detailed in Section 2.

Contribution. In this paper, a functional for the enhancement of gray-value images with the following advantages is proposed:

- intuitive control of the contrast level;

- choice of the spatial contrast scale;

- slight modification of the histogram.

The model is enlarged to color images such that

- the hue of the original image is approximately preserved.

In our model, the control of the level of contrast can be tuned with an intuitive parameter. A model with possible contrast level tuning is more adaptive to the image at hand. For instance, a binary image is useful for Optical Character Recognition, but it is not reliable in entertainment applications because it is unpleasant to watch.

A reliable enhancement has to be performed at a spatial scale defined by the user depending on its application. Since an image can be seen at multiple scales, the choice of this scale is significant for contrast enhancement. For example, the enhancement of the noise can be seen as a fine scale operation.

The proposed approach does not strongly modify the histogram of the original image. This enables the model to preserve the global lighting sensation. Moreover, as shown in the Checker Shadow Illusion of Adelson, the modification of the histogram is not local enough. Furthermore, if we consider an image with a large and uniform background, the modification of the histogram would damage it. In contrast, a large modification of the histogram is able to recover some details in an under-contrasted image.

The proposed model for color images preserves the hue of the image. The hue of the resulting image has to be as close as possible to the one of the input image. Indeed, the hue of an object does not change with the illumination.

Outline. In Section 2, we review models inspired by the HVS as the Retinex model and the model of Bertalmio et al. [7] which were the basis of our work. Then, in Section 3, we introduce a novel non-convex functional to enhance gray-scale images. Examples give some intuition on the idea behind the model. Furthermore, certain local minima are characterized. We present a minimization algorithm and demonstrate its performance by numerical examples. In Section 4 we start with some simple ideas how to generalize the above model to color images. Then, we merge the model with a color image enhancement method proposed by Fitschen et al. [19] within a variational framework. In contrast to the latter method, our new model does not require a given target intensity 
from some preprocessing step. The functional is then minimized by a hybrid primal-dual algorithm. Finally, comparisons with some state-of-the-art methods are performed on color images that demonstrate the efficiency of the proposed variational method.

\section{Models Inspired by the HVS}

We start by we recalling some state-of-the-art methods that were crucial for our work. Throughout this paper, we consider $M \times N$ digital images on the image grid

$$
\Lambda:=\{1, \ldots, M\} \times\{1, \ldots, N\}
$$

with values in $[0,1]$. Let $\mathcal{B}:=[0,1]^{M \times N}$.

Contrast enhancement can be performed by the Retinex model, developed by Land and McCann [31]. It states that the HVS does not perceive an absolute lightness but rather a relative, local one, see Figure 1. This phenomenon is called lateral inhibition. The aim of the original Retinex method is to remove the effect of the illumination over a scene. The noticeable work about Retinex by Jobson et al. [27] describes a so-called Single Scale Retinex algorithm. The Retinex output $\mathcal{R}(x)$ of the original image $u$ at pixel $x \in \Lambda$ is determined by

$$
\log (\mathcal{R}(x)):=\log (u(x))-\log ((G * u)(x)),
$$

where $G$ is a Gaussian kernel (frequently stated as surround function) and $*$ the convolution operator.

The Retinex model considers the formation of the image as a multiplicative process. The reflectance $\mathfrak{R}$ of the object is the light reflected by its own material. The illumination $L$ appears by smoothly illuminating the object by a light source. In the Retinex model, the light perceived by the observer (or a camera) is considered to be

$$
u(x)=L(x) \mathfrak{R}(x) .
$$

Assuming a certain smoothness of $L$, the value of interest $\mathfrak{R}$ is supposed to be well recovered by (1).

Method (1) corresponds to a multiplicative version of the ACE [20, 48, 49]. $\mathrm{ACE}$ is in particular based on the lateral inhibition. The ACE spatial chromatic comparison is defined as

$$
R(u)(x)=\sum_{y \in \Lambda} w(x, y) \varphi(u(x)-u(y)),
$$

where $\varphi$ is an increasing function such as the identity, a slope function, a sigmoid, or the sign function. The kernel $w$ has to be symmetric and to fulfill

$$
\sum_{y \in \Lambda} w(x, y)=1
$$

for all $x \in \Lambda$. The kernel models the lateral inhibition. More precisely, its size steers the spatial scale of the contrast enhancement. By removing the $\log$ operator in (1) we obtain the ACE term (3) with the identity function $\varphi$ and Gaussian weight $w$. Another assumption on the HVS, called gray-world 
hypothesis, is adopted in various variational models. It states that the mean of the perceived world is gray. Thus, the authors of [48] propose to average the image with the gray value $\frac{1}{2}$.

Based on the ACE findings, Bertalmio et al. [7] proposed an image enhancement method in a variational framework. It is related to Kernel Based Retinex models, see, e.g., [6] and makes use of the gray-world hypothesis. This method minimizes the following functional for each color channel $u$ of the image:

$$
\begin{aligned}
\frac{\alpha}{2} \sum_{x \in \Lambda}\left(u(x)-\frac{1}{2}\right)^{2}+\frac{\beta}{2} \sum_{x \in \Lambda}\left(u(x)-u_{0}(x)\right)^{2} & \\
& -\frac{1}{2} \sum_{x, y \in \Lambda} w(x, y) \Phi(u(x)-u(y)) .
\end{aligned}
$$

Here $u_{0} \in \mathcal{B}$ denotes the corresponding color channel of the original image, $\alpha$ and $\beta$ are non-negative parameters and $\Phi$ is a convex even function. The authors focus on $\Phi(t)=|t|$ which we will also be our choice in the rest of the paper. More general functions were examined in [40]. The first term pays attention to the gray-world hypothesis, the second term is the fidelity data term, and the third term, called average local contrast measure, favors contrasted images due to its negative sign. Since the functional is defined channel-wise, this model is not able to preserve the hue of the original image.

Using that the generalized gradient of the average local contrast measure is given by $R(u)$ in (3) with the sign function $\varphi$, the authors of [7] propose the following gradient descent algorithm to minimize (4):

$$
\begin{aligned}
& u^{(k+1)}=u^{(k)}- \\
& \Delta_{t}\left(\alpha\left(u^{(k)}-\frac{1}{2}\right)+\beta\left(u^{(k)}-u_{0}\right)-R u^{(k)}\right) \\
& =\left(1-(\alpha+\beta) \Delta_{t}\right) u^{(k)}+\Delta_{t}\left(\frac{\alpha}{2}+R\left(u^{(k)}\right)+\beta u_{0}\right) .
\end{aligned}
$$

Obviously, if $\left|u^{(k)}\right| \leq 1$, then $\left|R\left(u^{(k)}\right)\right| \leq 1$ componentwise. Thus, by induction, the stability of the system is guaranteed if $(\alpha+\beta) \Delta_{t}<1$ and $\alpha \geq 2$ because in this case

$$
\left|u^{(k+1)}\right| \leq 1-\Delta_{t}\left(\alpha+\beta-\frac{\alpha}{2}-\beta-1\right)=1-\Delta_{t}\left(\frac{\alpha}{2}-1\right) \leq 1 .
$$

In order to speed-up the evaluation of the operator $R$, the authors of [7] approximate the sign function by a polynomial. Appendix A details how this approximation accelerates the computation.

Unfortunately, the approximation by the sum of the Chebyshev polynomials proposed in [7] shows the typical Gibbs-like errors and takes values outside $[-1,1]$, see Figure 2. Thus, the approximation of $R$ remains no longer in $[-1,1]$. This leads to stability problems and indeed the algorithm can diverge with a high rate. To avoid this problem, the ACE implementation of [21] applies an affine mapping of the outcome of the $R$ operator towards $[-1,1]$ at each (or some) iteration. However, with this modification we have no longer a gradient descent algorithm, and to the best of our knowledge, the convergence of the sequence $\left\{u^{(k)}\right\}_{k}$ to a critical point of the functional is no longer guaranteed. 


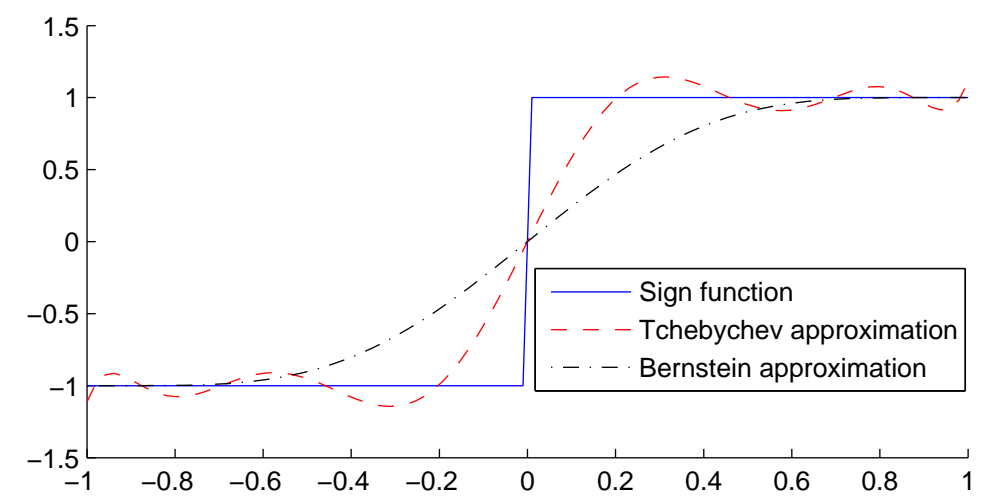

Figure 2: Bernstein and Chebyshev polynomial approximation of degree 9 of the sign function. The Bernstein approximation is bounded between -1 and 1 .

In order to stabilize the above numerical scheme without changing the model, the authors of [45] propose to replace the Chebyshev approximation by the sum of Bernstein polynomials so that $R$ stays within $[-1,1]$. The computation of the Bernstein approximation is detailed in Appendix A. Figure 2 shows the Bernstein and the Chebyshev approximations of the sign function.

Figure 3 shows the enhancement result of the method of Bertalmio et al. [7] with the Chebyshev approximation and affine rescaling and the Bernstein one without any additional mapping towards $[-1,1]$. The results are shown after 15 and 150 steps. Although both results after 150 steps are over-contrasted the one with the Chebyshev approximation is even worth.

Let us summarize some limitations of the reviewed perceptual model. The model is not hue preserving. The convergence of the gradient descent algorithm for model (4) is not guaranteed even if an additional affine rescaling is applied. In [40], Palma-Amestoy et al. demonstrate the convergence of the algorithm applied to the model (4) but with very small parameters $\alpha$ and $\beta$, not used in practical cases because the results would be over-contrasted. Hence the algorithm requires to limit the number of iterations. One may consider this as an advantage because a small number of iterations makes the performance faster, but the addition of new regularization terms may require another "optimal" number of iterations. In other words, the model is not flexible enough; the result depends on the number of iterations.

\section{Enhancement of Gray-Scale Images}

In this section, we propose a new model for the enhancement of gray-scale images. Its contrast level and spatial contrast tuning is intuitive.

We propose a projected gradient algorithm whose convergence to a critical point can be theoretically guaranteed. We demonstrate the very good performance of our method by numerical examples. 


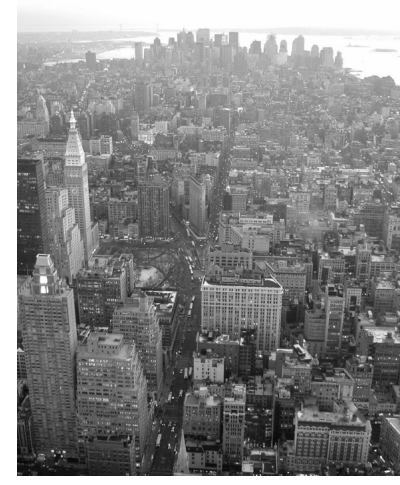

(a) Original image.

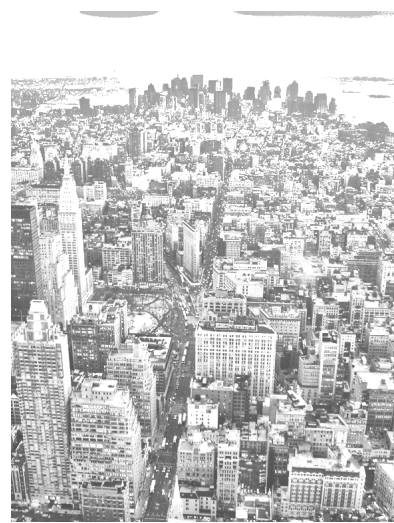

(d) Algorithm (5) with Cheby- (e) Algorithm (5) with Bernshev approximation [7] and 150 stein approximation and 150 ititerations.

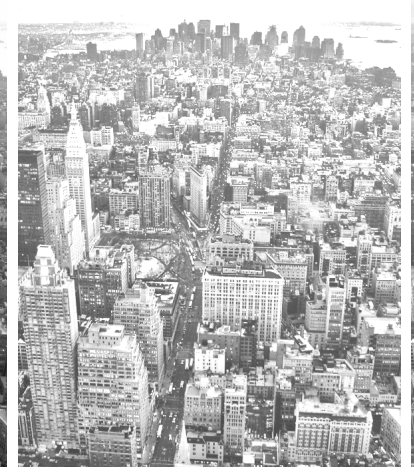

(b) Algorithm (5) with Cheby- (c) Algorithm (5) with Bernshev approximation [7] and 15 stein approximation and 15 ititerations.

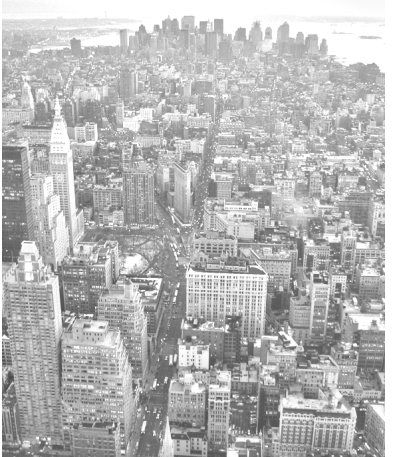

erations.

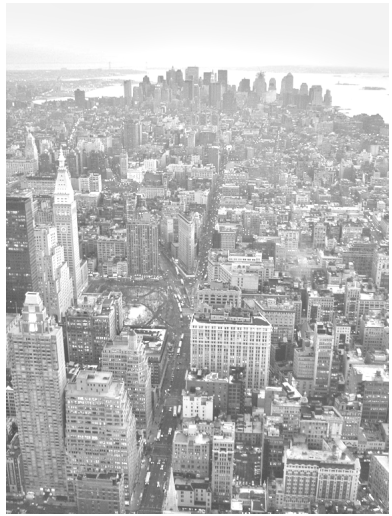
erations.

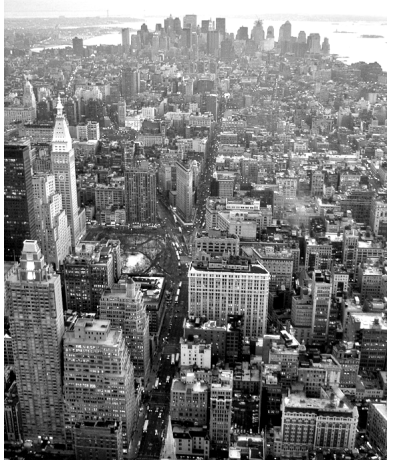

(f) Proposed approach.

Figure 3: Comparison between the algorithm of [7] with the Chebyshev polynomial approximation and the Bernstein one after 15 and 150 iterations. Too many iterations result in over-contrasted images. The proposed approach enables to control the contrast level.

\subsection{New Variational Model}

We restrict our attention to the discrete model and refer to Appendix B for the corresponding continuous one.

We use the average local contrast measure from (4) with the absolute value function $\Phi$ :

$$
\mathrm{C}(u):=\sum_{x \in \Lambda} \sum_{y \in \Lambda} w(x, y)|u(x)-u(y)| .
$$

The function $\mathrm{C}$ with a more general distance function is also known as Non-Local Total Variation, see, e.g., [5, 22].

Now our model for the enhancement of a given gray-scale image $u_{0} \in \mathcal{B}$ consists in minimizing the functional

$$
F(u):=\left\|u-u_{0}\right\|_{2}^{2}+\lambda\left(\mathrm{C}(u)-c \mathrm{C}\left(u_{0}\right)\right)^{2}+\iota_{\mathcal{B}}(u) .
$$


Here $\iota_{\mathcal{B}}$ denotes the indicator function of $\mathcal{B}$ which is zero within the set and $+\infty$ outside. It guarantees that the range of the image is preserved. The regularization parameter $\lambda>0$ manages the trade-off between the fidelity-data term and the contrast term. The constant $c>1$ implies that a minimizer of (8) increases the average local contrast measure. Intuitively, the contrast of the result tends to be $c$ times larger than the one of the input image. This can be interpreted as a multiplication by $c$ of the dynamic of the contours in the image. The choice of the parameter $c$ is intuitive and has a geometrical interpretation. It provides a control of the contrast level expected for the result. We give a simple illustrating example.

Example 1. (Role of the Contrast Value c)

Consider the discretized one-dimensional step function

$$
u_{0}:=\left(\frac{1}{4}, \frac{1}{4}, \frac{1}{4}, \frac{3}{4}, \frac{3}{4}, \frac{3}{4}\right)^{\mathrm{T}}
$$

depicted in Figure 4(a). We use the uniform kernel, i.e.,

$$
\mathrm{C}(u):=\sum_{x=1}^{6} \sum_{y=1}^{6} \frac{1}{6}|u(x)-u(y)| .
$$

Figure 4 shows the function $u_{0}$ as well as a local minimizer of the functional (8).

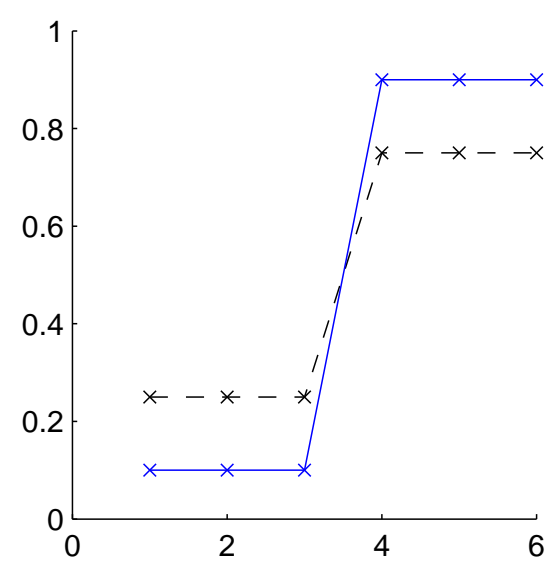

(a) Step function

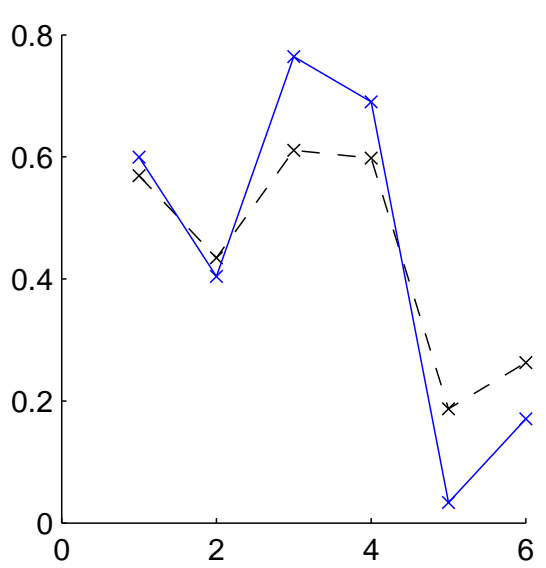

(b) Random function

Figure 4: Toy functions (dotted line) and a local minimizer of (8) (plain line). In each case, this minimizer looks like the original function, but its dynamic is improved, i.e., the difference between values of adjacent pixels is increased.

Note that the local minimizer is obtained by Proposition 1ii) in the next subsection and has the same relative pixel order as $u_{0}$. The local minimizer has the same shape as $u_{0}$. All differences between two adjacent pixels are increased, giving a higher dynamic to the result, and thus a higher contrast. This dynamic can be easily tuned modifying $c$. 
Example 2. (Role of the Kernel $w$ )

Let us consider the simple signal

$$
u_{0}(x)= \begin{cases}0 & \text { if } x \leq 1 / 3 \\ 1 / 2 & \text { if } x \in(1 / 3,2 / 3] \\ 1 & \text { if } x>2 / 3\end{cases}
$$

in Figure 5(a). This function contains two contours which have to be enforced. Since it is bounded between 0 and 1, it is impossible to increase the most left contour on its left-hand side. Thus, its right-hand side has to be increased in order to create an image with a higher average local contrast measure. The kernel function has a spatially limited effect. Assume that the spatial effect of the kernel is limited by its width of 0.2. Thus, the minimizer of the functional between 0.4 and 0.6 is mainly effected by the data fidelity term, and is indeed equal to $u_{0}$ here. A smooth transition is done between the two contours.

In the case of images, this smooth transition is roughly perceived as a constant part, when the width of the kernel is large enough. This phenomenon is related to the lateral inhibition. It is similar to the effect of the smoothness of the shadow in the Checker shadow illusion of Adelson in Figure 1. The width of the kernel has to be related to the size of the object of interest. This enables the proposed approach to be adapted to numerous applications.

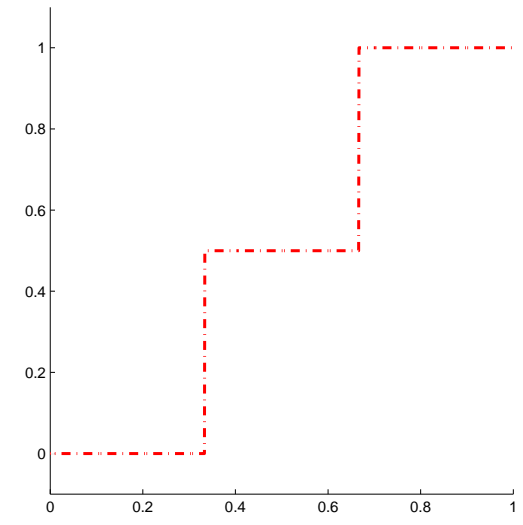

(a) Initial function (9)

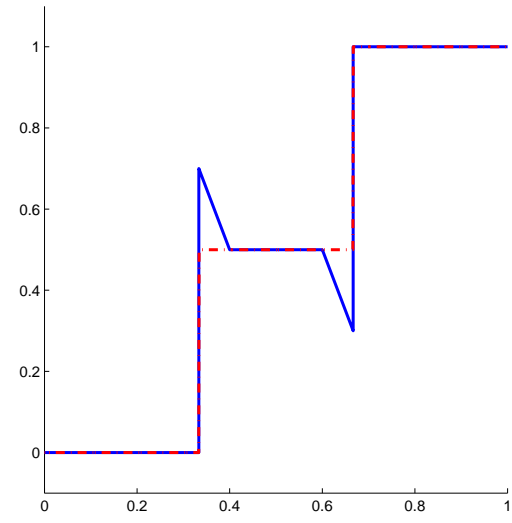

(b) Contrasted function

Figure 5: Original function (a) and contrasted one (b) with sharper contours.

Note that we do not impose a gray-world assumption for our model (8). On the one hand, it is often not fulfilled for natural images. On the other hand, this assumption enforces the result to be close to the histogram equalization. In contrast, we want to keep the histogram modification as small as possible, in order to preserve the global lighting sensation and handle large image areas having the same gray value. 


\subsection{Local Minima Characterization}

First note that $F: \mathbb{R}^{M \times N} \rightarrow[0,+\infty]$ in (8) is obviously proper, lower semicontinuous and coercive. Hence it has a global minimizer. However, the functional is not convex so that we are interested in local minima. The following proposition gives expressions for local minima under certain assumptions.

Theorem 1. i) If the relative order of the pixels of $u$ is known, then the average local contrast measure in (7) can be rewritten as

$$
\mathrm{C}(u)=2 \sum_{x \in \Lambda}\left(\sum_{y \in \Lambda} w(x, y) s_{u}(x, y)\right) u(x),
$$

with

$$
s_{u}(x, y):=\left\{\begin{aligned}
1 & \text { if } u(x)>u(y), \\
-1 & \text { if } u(x)<u(y), \\
0 & \text { if } u(x)=u(y) .
\end{aligned}\right.
$$

By reordering $u$ columnwise into a vector $\boldsymbol{u} \in \mathbb{R}^{n}, n=M N$, the average local contrast measure becomes

$$
\mathrm{C}(\boldsymbol{u})=V^{\mathrm{T}} \boldsymbol{u}
$$

with the vector $V=(V(x))_{x} \in \mathbb{R}^{n}$ given by

$$
V(x):=\sum_{y \in \Lambda} w(x, y) s_{u}(x, y) .
$$

ii) Assume that the relative order of $u$ and $u_{0}$ coincide. Then the functional $F$ in (8) can be written as

$$
F(\boldsymbol{u})=\left\|\boldsymbol{u}-\boldsymbol{u}_{0}\right\|_{2}^{2}+\lambda\left(V^{\mathrm{T}} \boldsymbol{u}-c V^{\mathrm{T}} \boldsymbol{u}_{0}\right)^{2}+\iota_{[0,1]^{n}}(\boldsymbol{u}) .
$$

If

$$
\hat{\boldsymbol{u}}:=\left(I d+\lambda V V^{\mathrm{T}}\right)^{-1}\left(I d+\lambda c V V^{\mathrm{T}}\right) \boldsymbol{u}_{0}
$$

has the same relative order as $\boldsymbol{u}_{0}$ and values in $[0,1]$, then $\hat{\boldsymbol{u}}$ is a local minimizer of $F$.

Proof. i) Due to the known order of $u$ we can rewrite the average local contrast measure as

$$
\begin{aligned}
C(u)= & \sum_{x \in \Lambda} \sum_{y \in \Lambda} w(x, y)|u(x)-u(y)| \\
= & \sum_{x \in \Lambda} \sum_{y \in \Lambda} w(x, y) s_{u}(x, y)(u(x)-u(y)) \\
= & \sum_{x \in \Lambda} \sum_{y \in \Lambda} w(x, y) s_{u}(x, y) u(x) \\
& -\sum_{x \in \Lambda} \sum_{y \in \Lambda} w(x, y) s_{u}(x, y) u(y)
\end{aligned}
$$

and since $s_{u}(x, y)=-s_{u}(y, x)$ further as

$$
C(u)=2 \sum_{x \in \Lambda}\left(\sum_{y \in \Lambda} w(x, y) s_{u}(x, y)\right) u(x) .
$$


This proves assertion $\mathrm{i}$ ).

ii) The vector form (10) of $F$ follows directly from i). The minimizer property of $\hat{\boldsymbol{u}}$ can be seen by setting the gradient of the first two summands of (10) to zero and verifying that its Hessian $I d+\lambda V V^{\mathrm{T}}$ is definite positive.

\subsection{Algorithm}

In this subsection, we propose an efficient algorithm for minimizing $F$ in (8). In order to apply a gradient-descent algorithm, the non-smooth contrast term is approximated by a polynomial. This polynomial approximation also enables us to speed-up the algorithm. The average local contrast measure $\mathrm{C}$ is approximated by

$$
\tilde{\mathrm{C}}(u)=\sum_{x, y \in \Lambda} w(x, y) \tilde{P}(u(x)-u(y)),
$$

where $\tilde{P}$ is an even polynomial which derivative $P=(\tilde{P})^{\prime}$ is a Bernstein polynomial approximation of the sign function explained in Appendix A. Note that

$$
\nabla \tilde{\mathrm{C}}(u)(x)=\sum_{y \in \Lambda} w(x, y) P(u(x)-u(y))
$$

which can be computed efficiently as outlined in the Appendix A Instead of $F$ we consider

$$
\tilde{F}(u):=\left\|u-u_{0}\right\|_{2}^{2}+\lambda\left(\tilde{\mathrm{C}}(u)-c \tilde{\mathrm{C}}\left(u_{0}\right)\right)_{2}^{2}+\iota_{\mathcal{B}}(u) .
$$

Since $\tilde{F}$ is composed of a differentiable part

$$
F_{\text {smooth }}(u):=\left\|u-u_{0}\right\|_{2}^{2}+\lambda\left(\tilde{\mathrm{C}}(u)-c \tilde{\mathrm{C}}\left(u_{0}\right)\right)_{2}^{2}
$$

and a box constraint, we propose to minimize it by the projected gradient algorithm detailed in Algorithm 1. Here $\Pi_{\mathcal{B}}$ denotes the orthogonal projection onto the convex set $\mathcal{B}$ and $\tau$ is the time step size which is fixed by Theorem 2 below. The efficient computation of $\nabla \tilde{\mathrm{C}}$ is described in Appendix A. The evaluation of $\tilde{\mathrm{C}}$ can be treated in a similar way.

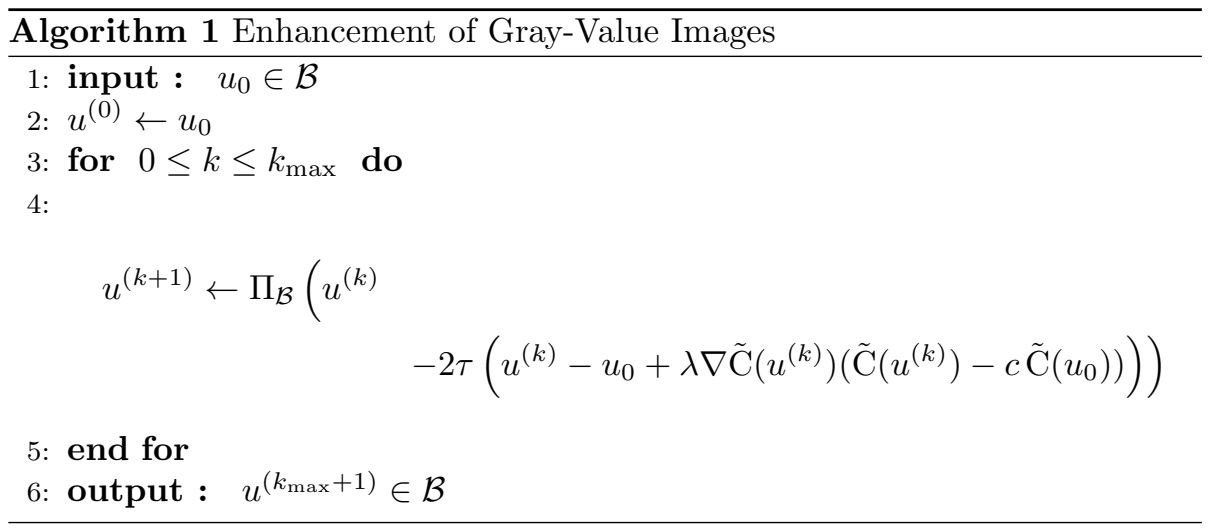

By the following theorem it can be at least ensured that the sequence $\left\{u^{(k)}\right\}_{k}$ generated by Algorithm 1 converges to critical point of $\tilde{F}$. 
Theorem 2. Let $L$ be the Lipschitz constant of the gradient of the function $F_{\text {smooth }}$ defined in $(13)$ on $\mathcal{B}$. Let $\epsilon \in\left(0, \frac{1}{2 L}\right)$. Then, for $\epsilon<\tau<\frac{1}{L}-\epsilon$, the sequence $\left\{u^{(k)}\right\}_{k}$ generated by Algorithm 1 converges to a critical point of $\tilde{F}$.

Proof. Since $F_{\text {smooth }}$ is polynomial in $u$, it is a real-analytic function. Moreover, as $\mathcal{B}$ is a non-empty closed semi-algebraic set, the function $\tilde{F}=F_{\text {smooth }}+\iota_{\mathcal{B}}$ is a semi-analytic function and therefore a Kurdyka-Lojasiewicz function [32]. Since the $u^{(k)}$ are bounded by construction, Theorem 5.3 of Attouch et al. [4] implies that Algorithm 1 converges to a critical point.

\subsection{Numerical Results}

We apply Algorithm 1 to gray-scale images. In practical cases, the value of $\tau$ is tuned manually to ensure the decreasing of the energy of the functional during the iterations. In all the presented cases, we used $\tau=10^{-6}$ and 20 iterations. Throughout this paper we will use the discretized Gaussian kernel with modifications at the boundary corresponding to mirror boundary conditions:

$$
w(x, y):=\frac{1}{\gamma} \mathrm{e}^{-\frac{\|x-y\|_{2}^{2}}{2 \sigma^{2}}}, \quad \gamma:=\sum_{x \in \mathbb{Z} \cap[-3 \sigma, 3 \sigma]} \mathrm{e}^{-\frac{\|x\|_{2}^{2}}{2 \sigma^{2}}} .
$$

Figure 6 provides results for various images. The details are better visible in the enhanced images and the image objects are better highlighted.

\section{Enhancement of Color Images}

Model (8), resp., (12) is designed to enhance gray-scale images. A simple generalization of our model to color images consists in treating each color channel separately. This method does not preserve the hue of the original image and can give non satisfactory results as in the Jupiter image in Figure 7 bottom, middle. The enhanced image is too shiny and has colors such as blue and purple, not present in the input image.

In this paper, we focus on color image enhancement that respects the hue of the original image. Recall that the hue of a single pixel $w=(r, g, b)$ is given by $H(w):=0$ if $r=g=b$ and otherwise by

$$
H(w):= \begin{cases}\theta & \text { if } b \leq g \\ 360^{\circ}-\theta & \text { if } b>g\end{cases}
$$

where

$$
\theta:=\arccos \frac{\frac{1}{2}((r-g)+(r-b))}{\left.\left((r-g)^{2}+(r-b)\right)(g-b)\right)^{\frac{1}{2}}},
$$

see [23]. Another possibility to enlarge our model (8) to color images is the two step algorithm described in the next subsection.

\subsection{Two Step Model for Color Image Enhancement}

Let $u_{0}:=\left(r_{0}, g_{0}, b_{0}\right), r_{0}, g_{0}, b_{0} \in \mathcal{B}$ be the given RGB image, i.e., the concatenation of $r, g$ and $b$ considered as three-dimensional array. In step 1, our model 

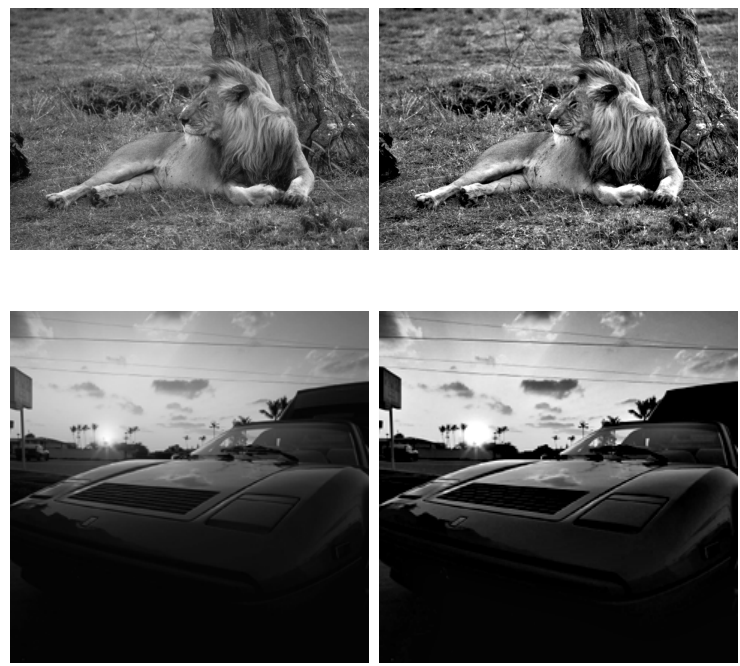

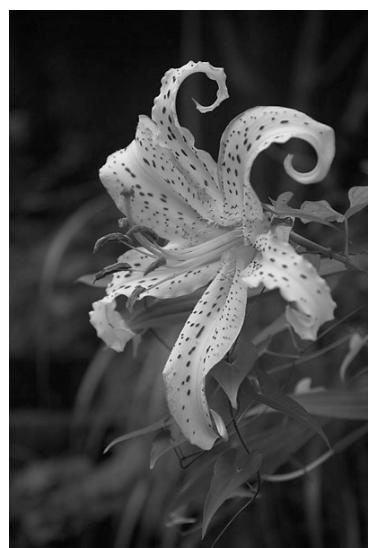

Original images.

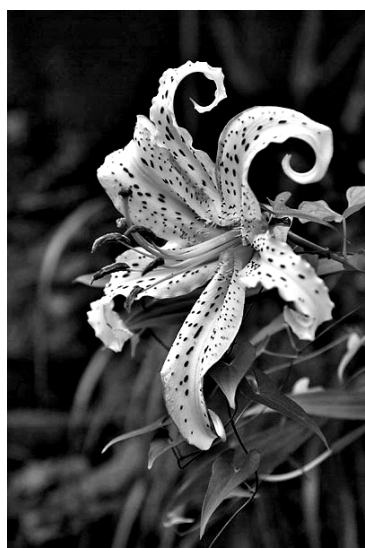

Enhanced images.

Figure 6: Results of Algorithm 1 on gray-scale images.

(8) is applied to the intensity image

$$
I_{0}:=\frac{1}{3}\left(r_{0}+g_{0}+b_{0}\right) \in \mathcal{B}
$$

which results in an enhanced intensity image $I \in \mathcal{B}$. Then, in a subsequent step, we apply the algorithm from $[35,36]$. More precisely, the so-called multiplicative method in [36] finds the new color image $u:=(r, g, b), r, g, b \in \mathcal{B}$ with prescribed intensity $I$ as follows: for every $x \in \Lambda$ set $M_{0}(x):=\max \left\{r_{0}(x), g_{0}(x), b_{0}(x)\right\}$. For every $\nu \in\{r, g, b\}$ compute

$$
\nu(x):= \begin{cases}\frac{\nu_{0}(x)}{I_{0}(x)} I(x) & \text { if } \frac{M_{0}(x)}{I_{0}(x)} I(x) \leq 1, \\ \frac{\nu_{0}(x)-I_{0}(x)}{M_{0}(x)-I_{0}(x)}(1-I(x)) & +I(x) \text { otherwise. }\end{cases}
$$

We set

$$
\frac{r_{0}(x)}{I_{0}(x)}=\frac{g_{0}(x)}{I_{0}(x)}=\frac{b_{0}(x)}{I_{0}(x)}:=1 \quad \text { if } \quad I_{0}(x)=0 .
$$


It is easy to check that the method preserves the range, i.e., $r, g, b \in \mathcal{B}$ and that $u:=(r, g, b)$ has the prescribed intensity

$$
I=\frac{1}{3}(r+g+b)
$$

By the following proposition which proof can be found, e.g., in [19] it follows immediately that the method (17) also preserves the hue.

Proposition 1. The single pixels $\left(r_{0}, g_{0}, b_{0}\right) \in[0,1]^{3}$ and $(r, g, b) \in[0,1]^{3}$ have the same hue if and only if there exist $a, d \in \mathbb{R}$ such that

$$
(r, g, b)=a\left(r_{0}, g_{0}, b_{0}\right)+d \mathbf{1}_{3}^{\mathrm{T}},
$$

where $\mathbf{1}_{3}:=(1,1,1)^{\mathrm{T}}$.

Figure 7 right shows that the two step method gives good results.

Let us mention that alternatively to the multiplicative algorithm in [36] its affine variant from the same paper, or variational methods as those in [44] can be applied in the second step. Indeed it was shown in [36] that for pixels treated by the first equation in (17), which are in general most of the image pixels, also the saturation of the original image is preserved. This leads to rather colorful images. Depending on the taste of the viewer this effect (colorfulness) can be steered using the affine model.
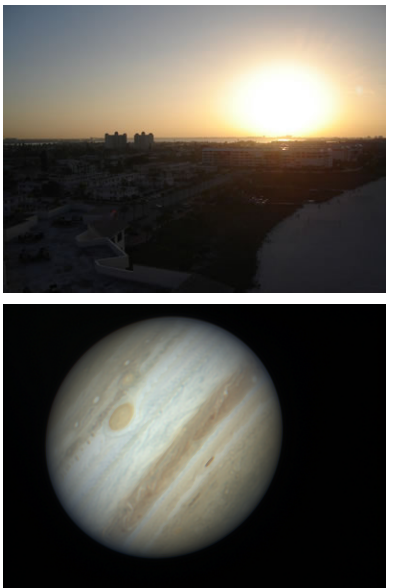

Original image
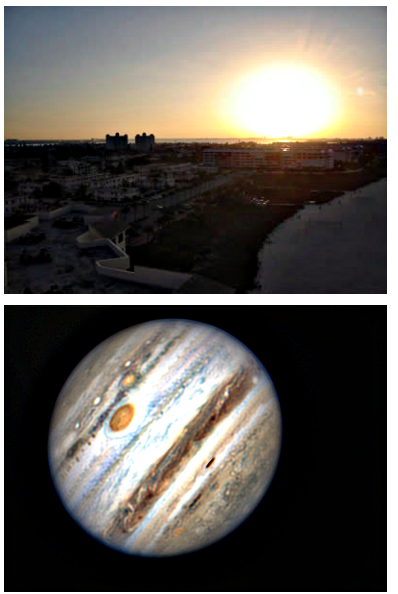

Channel-wise
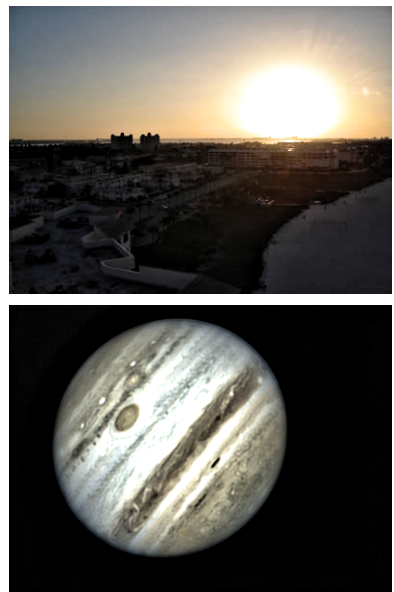

Intensity enhancement

Figure 7: Comparison of our method generalized to color images. First column: original image. Second column: channel-wise application. Third column: application to the intensity channel and subsequent application of the multiplicative algorithm in [36].

\subsection{New Variational Model}

In this section, we propose a model that enhances a given color image without a decoupling of the channels or a two step strategy. Our model preserves the hue approximately so that errors can be corrected. The idea consists in merging the 
model of Fitschen et al. [19] with the average local contrast measure from the previous section.

Given a color image $u_{0}$ with intensity $I_{0}$, the authors of [19] propose a variational model to obtain an enhanced image $u$ with prescribed intensity $I$ that respects to some degree the hue of the original image. To this end, auxiliary variables

$$
x_{R}, x_{G}, x_{B} \in \mathbb{R}^{M \times N} \text { and } d \in \mathbb{R}^{M \times N}
$$

are introduced which are related to the desired (concatenated) image $u=(r, g, b)$ by

$$
r:=\frac{r_{0}}{I_{0}} \circ x_{R}+d, g:=\frac{g_{0}}{I_{0}} \circ x_{G}+d, b:=\frac{b_{0}}{I_{0}} \circ x_{B}+d .
$$

Here the quotients

$$
A_{R}:=\frac{r_{0}}{I_{0}}, A_{G}:=\frac{g_{0}}{I_{0}}, A_{B}:=\frac{b_{0}}{I_{0}} \in \mathbb{R}^{M \times N}
$$

are meant componentwise, and $\circ$ denotes the componentwise (Hadamard) product. If $I_{0}(x)=0$, we use the setting in (18). To shorten the notation, we set

$$
x_{R G B}:=\left(x_{R}, x_{B}, x_{G}\right) \in \mathbb{R}^{M \times N \times 3}
$$

and denote the assignment in (20) by

$$
u=(r, g, b)=\left(A x_{R G B}, d\right) .
$$

Clearly, if $x_{A} \approx x_{G} \approx x_{B}$, then $\frac{x_{\nu}}{I_{0}}, \nu \in\{R, G, B\}$ in (20) approximate the constant $a$ in Proposition 1 so that $u$ should respect the hue of $u_{0}$. We enforce the approximate hue preservation by penalizing

$$
\tilde{H}\left(x_{R G B}\right):=\left\|x_{R}-x_{G}\right\|_{2}^{2}+\left\|x_{R}-x_{B}\right\|_{2}^{2}+\left\|x_{G}-x_{B}\right\|_{2}^{2} .
$$

The prescribed intensity $I$ is obtained if

$$
\begin{aligned}
I & =I\left(x_{R G B}, d\right) \\
& :=\frac{1}{3}\left(A_{R} \circ x_{R}+A_{G} \circ x_{G}+A_{B} \circ x_{B}\right)+d .
\end{aligned}
$$

To cope with noise while preserving the image edges we will exploit the discrete total variation (TV) [50] of $u$. We define the discrete gradient of the color channels $\nu \in\{r, g, b\}$ at $x=(i, j) \in \Lambda$ by

$$
\begin{aligned}
& \nabla \nu(x):=\left(\begin{array}{c}
D_{x} \nu(x) \\
D_{y} \nu(x)
\end{array}\right) \\
& =\left(\begin{array}{rl}
\nu(i+1, j)-\nu(i, j) & \text { if } i<N, \\
0 \text { if } i=N & , \\
\nu(i, j+1)-\nu(i, j) & \text { if } j<M, \\
0 \text { if } j=M & ,
\end{array}\right) .
\end{aligned}
$$


Then the total variation of $u$ in terms of $x_{R G B}$ and $d$ is given by

$$
\begin{aligned}
T V\left(x_{R G B}, d\right):= & \sum_{x \in \Lambda}\left(\sum_{\nu \in\{r, g, b\}} D_{x} \nu(x)^{2}+D_{y} \nu(x)^{2}\right)^{\frac{1}{2}} \\
= & \sum_{x \in \Lambda}\left(\sum_{\nu \in\{R, G, B\}} D_{x}\left(A_{\nu} \circ x_{\nu}+d\right)(x)^{2}\right. \\
& \left.+D_{y}\left(A_{\nu} \circ x_{\nu}+d\right)(x)^{2}\right)^{\frac{1}{2}}
\end{aligned}
$$

Taking (22), (24) and (23) into account, and demanding the range preservation in (21), the authors of [19] propose the following model:

$$
\begin{gathered}
\underset{x_{R G B}, d}{\arg \min }\left\{\frac{1}{2}\|d\|_{2}^{2}+\frac{\mu}{2} \tilde{H}\left(x_{R G B}\right)+\lambda \operatorname{TV}\left(x_{R G B}, d\right)\right\} \\
\text { subject to } \quad\left\{\begin{array}{l}
\left(A x_{R G B}, d\right) \in \mathcal{B}^{3}, \\
I=I\left(x_{R G B}, d\right),
\end{array}\right.
\end{gathered}
$$

where $\lambda, \mu \geq 0$. If $\hat{x}_{R G B}, \hat{d}$ is a solution, then $\left(A \hat{x}_{R G B}, \hat{d}\right)$ is the desired image. Note that the squared norm of $d$ is penalized to enforce the model to have a minimizer which is near to the multiplicative model (17). Indeed, it was shown in [19] that for large $\mu$ and small $\lambda$ the solution in (17) is obtained. However, model (25) still requires that an enhanced intensity image $I$ is known.

We merge the above variational model with model (8) to get a unified variational framework for color image enhancement without pre-assigning a well contrasted intensity image. Our new model reads as follows:

$$
\begin{aligned}
\underset{x_{R G B}, d}{\arg \min } & \left\{\frac{\lambda}{2}\|d\|_{2}^{2}+\frac{\mu}{2} \tilde{H}\left(x_{R G B}\right)+\operatorname{TV}\left(x_{R G B}, d\right)\right. \\
& +\frac{\alpha}{2}\left(\tilde{\mathrm{C}}\left(I\left(x_{R G B}, d\right)\right)-c \tilde{\mathrm{C}}\left(I_{0}\right)\right)^{2} \\
& \left.+\frac{\beta}{2}\left\|I\left(x_{R G B}, d\right)-I_{0}\right\|_{2}^{2}\right\} \\
& \text { subject to }\left(A x_{R G B}, d\right) \in \mathcal{B}^{3} .
\end{aligned}
$$

The aim of this model is to enhance at the same time the intensity channel and to assign to each pixel a color which hue is close to that of the original one. A high value of $\mu$ ensures that the hue of the new image is close to the original one. We penalize the Total Variation in order to remove noisy pixels or artifacts. The scale of the contrast enhancement is controlled by the size of the kernel used for the computation of $\tilde{\mathrm{C}}$. The contrast level is tuned by the parameter $c$. The parameter $\lambda$ enforces the algorithm towards the multiplicative method (17).

\subsection{Algorithm}

Chambolle and Pock [14] propose an algorithm to compute a saddle-point of a problem of the form

$$
\min _{p} \max _{q}\langle K p, q\rangle+f(p)+g(p)-h^{*}(q)
$$


where $f$ has a Lipschitz gradient with constant $L_{f}$, and $g, h$ are proper, lower semi-continuous, convex functions with Fenchel dual

$$
h^{*}(q)=\sup _{y}\langle q, y\rangle-h(y) .
$$

Further, $K$ is a bounded linear operator with operator norm $L=\|K\|$. For former versions of primal-dual algorithms see also [13, 46, 57]. The general iteration of the algorithm

$$
(\hat{p}, \hat{q})=P D(\bar{p}, \bar{q}, \tilde{p}, \tilde{q})
$$

is defined by

$$
\begin{gathered}
\hat{p}=\underset{p}{\arg \min }\{f(\bar{p})+\langle\nabla f(\bar{p}), p-\bar{p}\rangle+g(p)+\langle K p, \tilde{q}\rangle \\
\left.+\frac{1}{2 \tau}\|p-\bar{p}\|_{2}^{2}\right\} \\
\hat{q}=\underset{q}{\arg \min }\left\{h^{*}(q)-\langle K \tilde{p}, q\rangle+\frac{1}{2 \sigma}\|q-\bar{q}\|_{2}^{2}\right\}
\end{gathered}
$$

The authors of [14] propose to use the iteration

$$
\left(p^{(k+1)}, q^{k+1}\right)=P D\left(p^{(k)}, q^{(k)}, 2 p^{(k+1)}-p^{(k)}, q^{(k)}\right),
$$

and prove the convergence of the algorithm if

$$
\left(\frac{1}{\tau}-L_{f}\right) \frac{1}{\sigma} \geq L^{2} .
$$

In order to apply the algorithm to our model (26), we reshape all images from $\mathbb{R}^{M \times N}$ columnwise into vectors of length $n=M N$. This is often described by the vec operation. Since the format becomes clear from the context we keep the notation $x$ instead of $\operatorname{vec}(x)$. In particular, we have $x_{R G B} \in \mathbb{R}^{3 n}$ and $d \in \mathbb{R}^{n}$ now. Moreover, after reshaping $A_{\nu}, \nu \in\{R, G, B\}$ into vectors of length $n$, we built the diagonal matrices $\boldsymbol{A}_{\nu}, \nu \in\{R, G, B\}$ out of these vectors. We can rewrite the total variation term for this setting by applying the vector adapted gradient operator $\nabla: \mathbb{R}^{n} \mapsto \mathbb{R}^{2 n}$ below

$$
\boldsymbol{\nabla}:=\left(\begin{array}{l}
I_{N} \otimes D_{M} \\
D_{N} \otimes I_{M}
\end{array}\right), \quad D_{M}:=\left(\begin{array}{cccc}
-1 & 1 & & \\
& \ddots & \ddots & \\
& & -1 & 1 \\
& & & 0
\end{array}\right) \in \mathbb{R}^{M, M},
$$

where $\otimes$ denotes the Kronecker product and $I_{N}$ is the $N \times N$ identity matrix. Setting

$$
\begin{aligned}
f\left(x_{R G B}, d\right):= & \frac{\lambda}{2}\|d\|_{2}^{2}+\frac{\mu}{2} \tilde{H}\left(x_{R G B}\right) \\
& +\frac{\alpha}{2}\left(\tilde{\mathrm{C}}\left(I\left(x_{R G B}, d\right)\right)-c \tilde{\mathrm{C}}\left(I_{0}\right)\right)^{2} \\
& +\frac{\beta}{2}\left\|I\left(x_{R G B}, d\right)-I_{0}\right\|_{2}^{2}, \\
g\left(x_{R G B}, d\right):= & 0, \\
h(v, u):= & \|v\|_{2,1}+\iota_{[0,1]^{3 n}}(u)
\end{aligned}
$$


and

$$
K:=\left(\begin{array}{c|c}
\left(I_{3} \otimes \boldsymbol{\nabla}\right) \boldsymbol{A} & \mathbf{1}_{3} \otimes \boldsymbol{\nabla} \\
\hline \boldsymbol{A} & \mathbf{1}_{3} \otimes I_{n}
\end{array}\right), \quad \boldsymbol{A}:=\left(\begin{array}{ccc}
\boldsymbol{A}_{R} & & \\
& \boldsymbol{A}_{G} & \\
& & \boldsymbol{A}_{B}
\end{array}\right),
$$

our model (26) can be rewritten as

$$
\begin{aligned}
\min _{\substack{x_{R G B}, d \\
v, u}}\left\{f\left(x_{R G B}, d\right)+h(u, v)\right\} \\
\text { subject to } K\left(\begin{array}{c}
x_{R G B} \\
d
\end{array}\right)=\left(\begin{array}{c}
v \\
u
\end{array}\right) .
\end{aligned}
$$

For $v \in \mathbb{R}^{6 n}$ we have used the norm

$$
\|v\|_{2,1}:=\sum_{i=1}^{n}\left(\sum_{j=1}^{6} v_{i+j n}^{2}\right)^{\frac{1}{2}} .
$$

The above model (30) is equivalent to

$$
\begin{aligned}
\min _{\substack{x_{R G B}, d \\
v, u}} \max _{q_{1}, q_{2}}\left\{f\left(x_{R G B}, d\right)+h(u, v)\right. \\
\left.+\left\langle K\left(\begin{array}{c}
x_{R G B} \\
d
\end{array}\right)-\left(\begin{array}{c}
v \\
u
\end{array}\right),\left(\begin{array}{l}
q_{1} \\
q_{2}
\end{array}\right)\right\rangle\right\} .
\end{aligned}
$$

Using that $\min _{v, u} \varphi=-\max _{v, u}(-\varphi)$, changing the order of $\max _{v, u}$ and $\max _{q_{1}, q_{2}}$ and applying the definition of the Fenchel dual, our model obtains finally the form of (27):

$$
\begin{aligned}
\min _{x_{R G B}, d} \max _{q_{1}, q_{2}}\left\{f\left(x_{R G B}, d\right)-h^{*}\left(q_{1}, q_{2}\right)\right. \\
\left.+\left\langle K\left(\begin{array}{c}
x_{R G B} \\
d
\end{array}\right),\left(\begin{array}{c}
q_{1} \\
q_{2}
\end{array}\right)\right\rangle\right\} .
\end{aligned}
$$

Then the first part (28) of the algorithm reads

$$
\begin{aligned}
\hat{p} & =\underset{p}{\arg \min }\left\|p-\bar{p}+\tau\left(\nabla f(\bar{p})+K^{*} \tilde{q}\right)\right\|_{2}^{2} \\
& =\bar{p}-\tau\left(\nabla f(\bar{p})+K^{*} \tilde{q}\right) .
\end{aligned}
$$

and regarding that

$$
K^{*}:=\left(\begin{array}{c|c}
\boldsymbol{A}\left(I_{3} \otimes \boldsymbol{\nabla}^{\mathrm{T}}\right) & \boldsymbol{A} \\
\hline \mathbf{1}_{3}^{\mathrm{T}} \otimes \boldsymbol{\nabla}^{\mathrm{T}} & \mathbf{1}_{3}^{\mathrm{T}} \otimes I_{n}
\end{array}\right)
$$

we obtain Step 7 and 8 in Algorithm 2. The computation of $\nabla f$ is outlined in Remark 1. Note that $\nabla^{\mathrm{T}}$ plays the role of the negative divergence operator in the continuous setting.

Concerning the second part (29) of the algorithm, we have

$$
\begin{aligned}
h^{*}\left(q_{1}, q_{2}\right) & =\left(\|\cdot\|_{2,1}\right)^{*}\left(q_{1}\right)+\iota_{[0,1]^{3 n}}^{*}\left(q_{2}\right) \\
& =\iota_{B_{2, \infty}}\left(q_{1}\right)+q_{2} \chi \geq 0
\end{aligned}
$$


where $B_{2, \infty}$ denotes the unit ball with respect to the dual norm of the $\ell_{2}-\ell_{1}$ norm

$$
\|v\|_{2, \infty}:=\max _{i=1 \ldots, n}\left(\sum_{j=1}^{6} v_{i+j n}^{2}\right)^{\frac{1}{2}},
$$

and $\chi \geq 0$ is the component-wise characteristic function of the non negative numbers, i.e., it is one for non negative numbers and zero otherwise. Then we can rewrite (29) as

$$
\begin{aligned}
\hat{q} & =\underset{q}{\arg \min } h^{*}(q)+\frac{1}{2 \sigma}\left\|q-\bar{q}-\sigma K\left(\tilde{x}_{R G B}, \tilde{d}\right)^{\mathrm{T}}\right\|_{2}^{2}, \\
\hat{q}_{1} & =\Pi_{B_{2, \infty}}\left(\bar{q}_{1}+\sigma\left(\left(I_{3} \otimes \boldsymbol{\nabla}\right) \boldsymbol{A} \tilde{x}_{R G B}+\left(\mathbf{1}_{3} \otimes \boldsymbol{\nabla}\right) \tilde{d}\right)\right) \\
\hat{q}_{2} & =\sigma\left(y-\Pi_{[0,1]^{3 n}}(y)\right), \\
y & :=\frac{1}{\sigma} \bar{q}_{2}+\boldsymbol{A} \tilde{x}_{R G B}+\left(\mathbf{1}_{3} \otimes I_{n}\right) \tilde{d} .
\end{aligned}
$$

This is used in Step 11-13 of Algorithm 2.

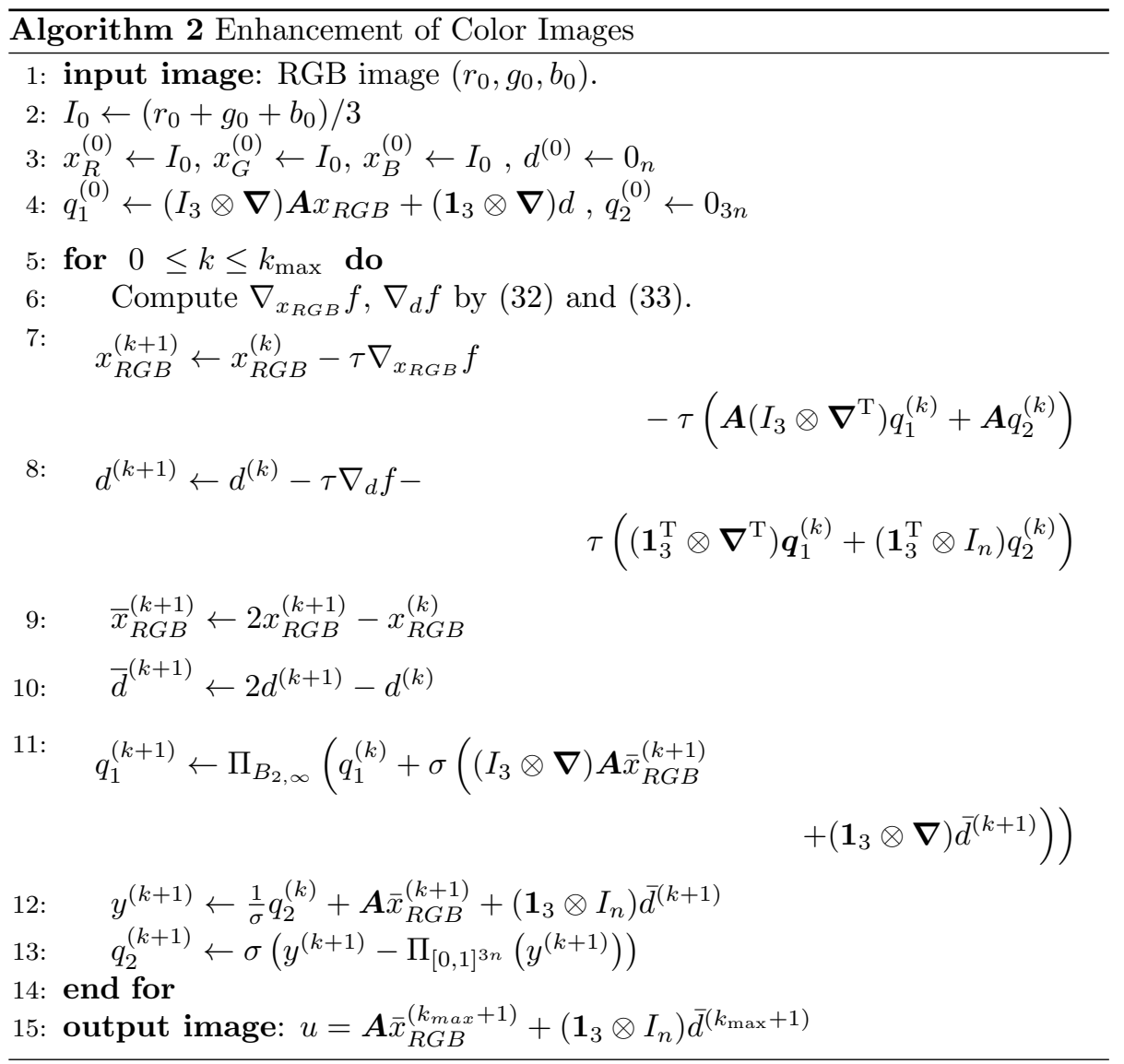

The most expensive computation is the one of $\tilde{\mathrm{C}}$ and $\nabla \tilde{\mathrm{C}}$ embedded in the computation of $\nabla f$. This computation is accelerated in the same way as for Algorithm 1, see Appendix A. 
Remark 1. (Computation of $\nabla f=\nabla_{x_{R G B}} f, \nabla_{d} f$ )

The derivative of $f$ with respect to $x_{\nu}, \nu=R, G, B$ is given by

$$
\begin{aligned}
\frac{\partial f}{\partial x_{\nu}}=\mu\left(2 x_{\nu}-x_{\hat{\nu}}-x_{\tilde{\nu}}\right) & \\
& +\frac{\alpha \nu_{0}}{3 I_{0}} \nabla \tilde{\mathrm{C}}\left(I\left(x_{R G B}, d\right)\right)\left(\tilde{\mathrm{C}}\left(I\left(x_{R G B}, d\right)\right)-c \tilde{\mathrm{C}}\left(I_{0}\right)\right) \\
& +\frac{\beta \nu_{0}}{3 I_{0}}\left(I\left(x_{R G B}, d\right)-I_{0}\right),
\end{aligned}
$$

where $\tilde{\nu}, \hat{\nu} \in\{R, G, B\}$. The derivative of $f$ with respect to $d$ reads

$$
\begin{aligned}
\frac{\partial f}{\partial d}=\lambda d+\alpha \nabla \tilde{\mathrm{C}}\left(I\left(x_{R G B}, d\right)\right)\left(\tilde{\mathrm{C}}\left(I\left(x_{R G B}, d\right)\right)-\right. & \left.c \tilde{\mathrm{C}}\left(I_{0}\right)\right) \\
& +\beta\left(I\left(x_{R G B}, d\right)-I_{0}\right) .
\end{aligned}
$$

The convergence of Algorithm 2 is not guaranteed because model (26) is not convex, but in practice, numerical convergence was observed.

Finally, let us emphasize that we use the Kronecker product notation only for a correct description of the algorithm. In our numerical computations we work with arrays based on the relation

$$
\operatorname{vec}\left(A X B^{\mathrm{T}}\right)=(B \otimes A) \operatorname{vec}(X) .
$$

\subsection{Numerical Results}

In this section, Algorithm 2 is applied to color images and the results are compared to some state-of-the-art methods. The algorithm is implemented in MATLAB.

The following parameters have been experimentally chosen: $\lambda=100, \mu=1$, $\alpha=100, \beta=500, \sigma=5.10^{-5}, \tau=5.10^{-9}$ and $10^{3}$ iterations. The polynomial approximation is done with Bernstein polynomials of degree 9. The size of the Gaussian kernel used for $\mathrm{C}$ has to be related to the size of the image. In experiments it is experimentally chosen equal to $\min (M, N) / 20$. This size manages the scale of the contrast enhancement. Its choice is discussed at the end of this subsection.

In Figure 8, we compare our method to the equalization implementation of Gimp, the algorithm of Nikolova et al. [37, 36] with implementation [26], the method of Bertalmio et al. [7] and the one of Ferradans et al. [18]. For the comparison approaches, we used the default parameters of the papers. The implementation of Gimp consists in a histogram equalization of the intensity channel. This method is not able to preserve the hue, because the transformation from the HSV color-space to RGB can change the hue due to the Gamut problem (see, e.g., [44]).

In the Jupiter image, the approach of $[7,18]$ such as the one of the Gimp performs a modification of the histogram. However, it contains a peak, corresponding to the background. Thus, an important modification of the histogram is not useful for this type of images. Tackling with this issue, the proposed approach preserves the histogram and thus the background.

In the Chandelier image, the method of [7], the approach of [18] as well as the one of Gimp change the color of the ceiling due the lack of hue preservation. 

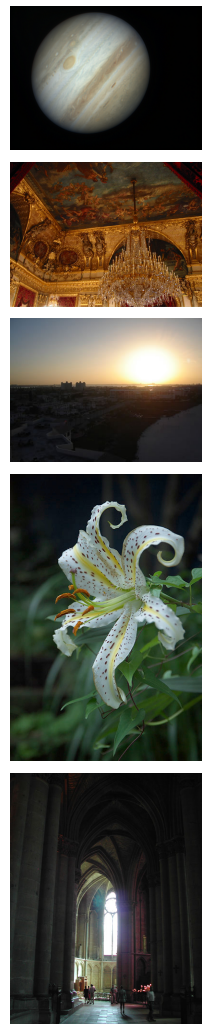

Original
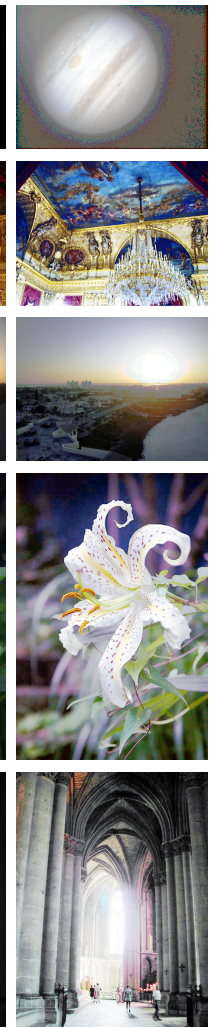

Gimp
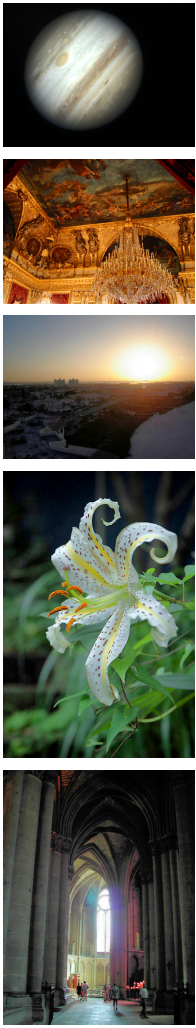

[36]
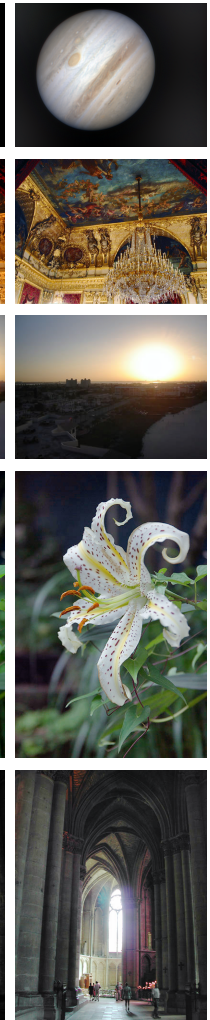

[7]
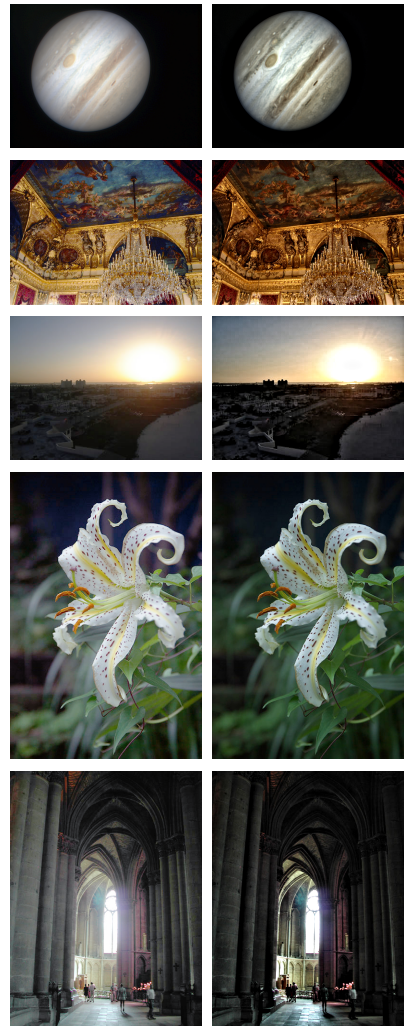

[18]

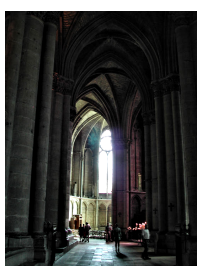

Our

Figure 8: Comparison of our enhancement results with those obtained by the algorithms of the Gimp histogram equalization, of Nikolova et al. [36], of Bertalmio et al. [7], of Ferradans et al. [18]. The proposed algorithm produces good results in all these cases.

In contrast, the proposed approach, as well as the one of [36], produces results with nearly the original hue. The preservation of the hue is needed to obtain these results, and may be important in some application such as art restoration.

For the Sunrise image, only Algorithm 2 is able to produce an image on which the building in the center is clearly visible. In the other images, the results are over-contrasted, in particular near the sun. With the controlled contrast level, the proposed method is able to recover the desired informations.

For the Iris image, the method of $[7,18]$ and the one of Gimp provide images with a purple background due the modification of the hue. The approach of $[37,36,26]$ respects the hue. The proposed algorithm controls the contrast level and respects the hue. Thus it is thus able to enforce the visibility of the leaf veins.

Finally, in the Cathedral image, the enhanced details are different for each method. State-of-the-art methods enhance the details in the foreground contrary to the proposed approach which enforces the background. The lighting sensation is also different on the different approaches: for all comparison approaches, it seems that the light is behind the photographer in order to en- 


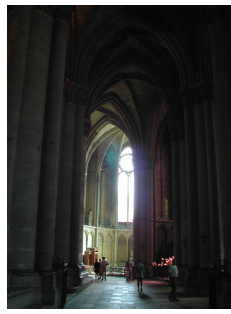

Original

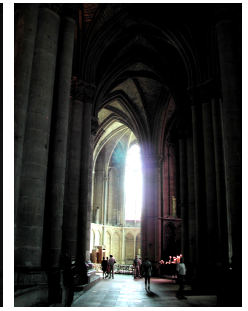

Coarse scale Medium scale Fine scale $(c=$

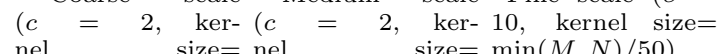
$\min (M, N) / 2) \quad \min (M, N) / 20)$

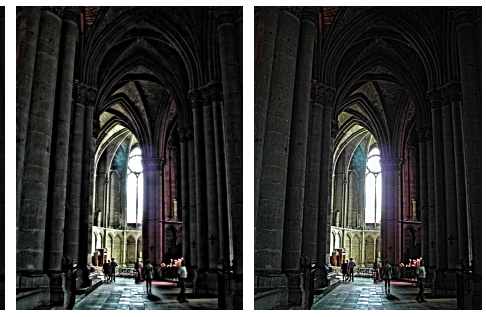

Very fine scale
kernel $(c=\underset{\text { size }}{10}=$ $\min (M, N) / 120)$

Figure 9: Influence of the contrast enhancement scale on the result. This scale has to be tuned in dependence on the desired result.

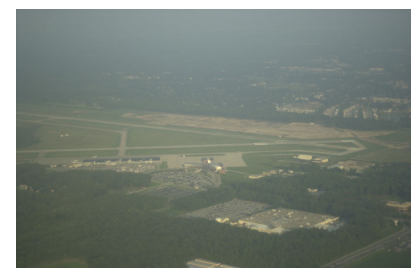

Original

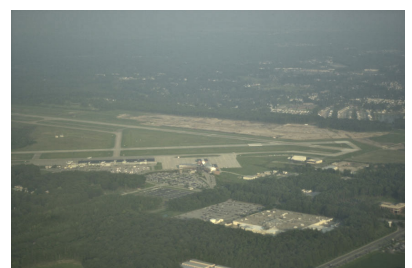

Medium level and coarse High level and fine scale $(c=10$ scale $(c)=2$ size $=\min (M, N) / 20)$

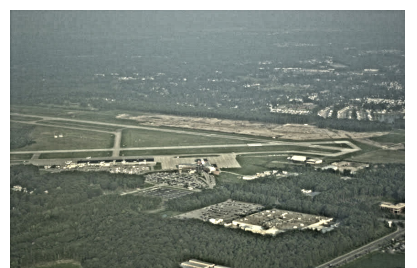

kernel kernel $\operatorname{size}=\min (M, N) / 50)$

Figure 10: Influence of the contrast enhancement scale and level on the result. In function of the application, these parameters have to be tuned.

lighten the columns of the Cathedral, contrary to our result that preserves the light sensation and the shadows of the scene. These facts are due to the contrast scale. In the following, we show that a modification of the contrast scale of the algorithm may change the location of the enhanced details such as the lighting sensation and the shadows. Figure 11 proposes some additional results obtained with Algorithm 2.

Importance of the contrast enhancement scale. For the Cathedral image on Figure 8, our result appears to be less contrasted than the others in some parts of the image. Some details near the windows are better visible than with the others methods, but some details are not visible, for instance the details in the shadows of the columns. This is due to the scale of the enhancement. By changing this scale, our method can recover different details, as shown in Figure 9. All the enhanced images do not have the same contextual information. For instance, at coarse scale, the overall lighting of the scene is enhanced. The over-lighting of the window is increased. For the medium scale, the shadows are enforced and the details close to the window are more visible. The overall lighting of the scene is farther from the original image than at the coarse scale. With the finest scale, the shadows are less enforced, but the details of the textures of the column are enhanced. The tuning of the contrast scale depends on the targeted application. A trade-off has to be chosen by the user between a 


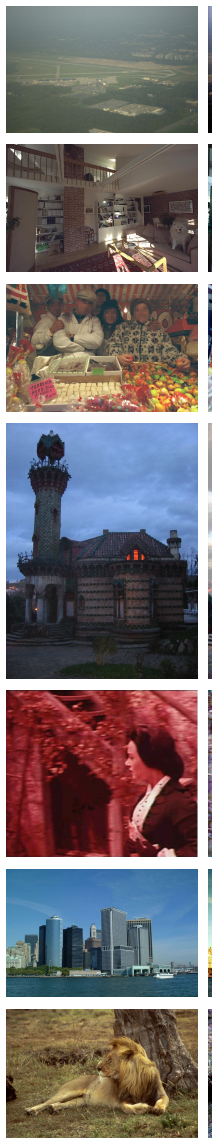

Original
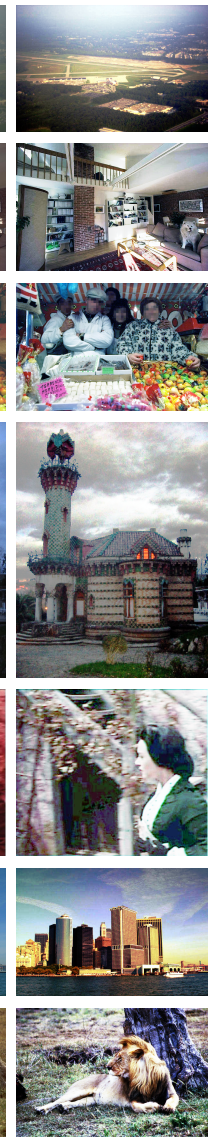

Gimp
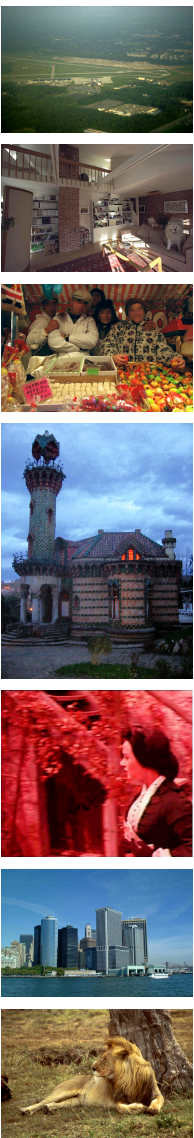

[36]
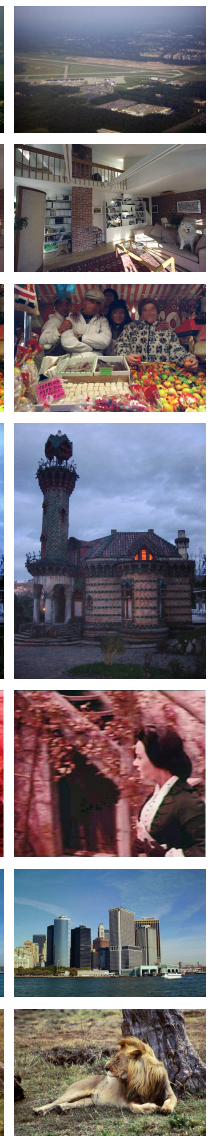

[7]
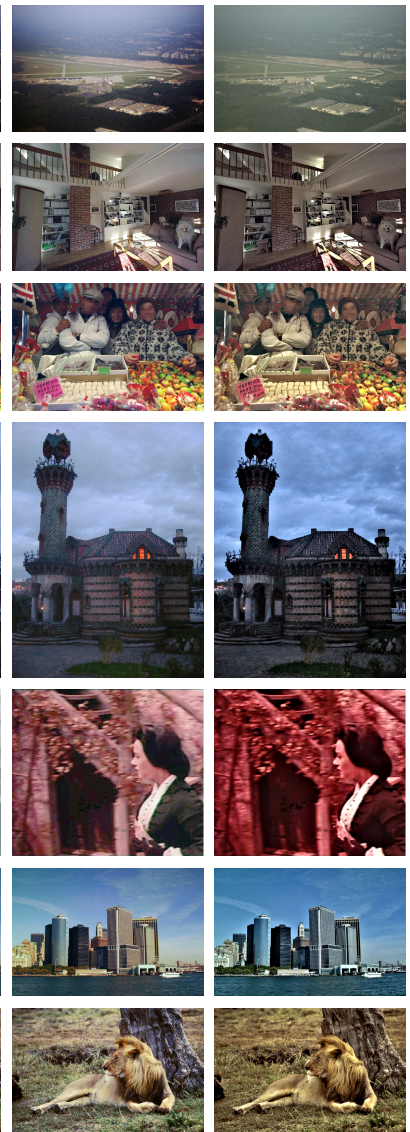

[18]

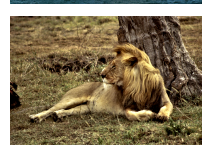

Our

Figure 11: Additional results.

preservation of the lighting sensation and the expected details. Figure 10 shows the performance of Algorithm 2 for different contrast levels and scales. At a large scale and low contrast level, the model is able to produce an image with more details, but with the same lighting sensation as the original one, i.e., a hazed aerial photography. At a fine contrast scale and a high contrast level, the model is able to provide an image which can be used, for example, to detect the number of planes on the airfield. As a conclusion, depending on the application, a good parameter tuning makes our model fully adaptive.

\section{Summary and Conclusions}

We introduced variational models both for the enhancement of gray-scale images and color images. We show that increasing the average local contrast measure improves the perceived contrast of the image. The convergence of the minimization algorithm for gray-scale images is proved. The contrast scale and level in our model are adjustable, so that the proposed approach is fully adaptive. Our enhancement method for color images works directly on the RGB image without 
decoupling of the color channels or enhancing the intensity in a previous step. We demonstrated that our model is able to enhance contrast on images where state-of-the-art methods fail. The automatic adaptation of the parameters to the content of the considered image could be a future direction of research.

\section{A Efficient Computation by Polynomial Approx- imation}

First we show how the sign function can be approximated by Bernstein polynomials. The Bernstein polynomial on $[0,1]$ of degree $n$ is defined by

$$
B_{k}^{n}(t):=\left(\begin{array}{l}
n \\
k
\end{array}\right) t^{k}(1-t)^{n-k} .
$$

By Weistraß' theorem, any continuous function $f:[0,1] \rightarrow \mathbb{R}$ can be approximated by

$$
b_{n}(f, t)=\sum_{k=0}^{n} f\left(\frac{k}{n}\right) B_{k}^{n}(t)
$$

and $\left\|f-b_{n}(f, \cdot)\right\|_{\infty}$ goes to zero as $n \rightarrow \infty$. We approximate the jump function $g:[0,1] \rightarrow \mathbb{R}$ defined by

$$
g(t):= \begin{cases}0 & \text { if } t \in\left[0, \frac{1}{2}\right), \\ \frac{1}{2} & \text { if } t=\frac{1}{2} \\ 1 & \text { if } t \in\left(\frac{1}{2}, 1\right]\end{cases}
$$

and use $P(t):=2 b_{n}\left(g, \frac{t+1}{2}\right)-1$ as an approximation of the sign function. Next, we write $b_{n}\left(g, \frac{t+1}{2}\right)$ as the sum of monomials

$$
\left(\frac{1}{2}\right)^{n} \sum_{k=0}^{n}\left(\begin{array}{l}
n \\
k
\end{array}\right) g\left(\frac{k}{n}\right) \sum_{i=0}^{k} \sum_{j=0}^{n-k}\left(\begin{array}{c}
k \\
i
\end{array}\right)\left(\begin{array}{c}
n-k \\
j
\end{array}\right)(-1)^{j} t^{i+j}
$$

Having a polynomial representation $P(t)=\sum_{m=0}^{n} c_{m} t^{m}$ available, we can apply an idea from [7] in order to efficiently compute $\nabla \tilde{C}(u)(x)$ if $w(x, y)=G(x-y)$ :

$$
\begin{aligned}
\nabla \tilde{C}(u)(x) & =\sum_{y \in \Lambda} w(x, y) \sum_{m=0}^{n} c_{m}(u(x)-u(y))^{m} \\
& =\sum_{y \in \Lambda} w(x, y) \sum_{m=0}^{n} c_{m}(-1)^{k} \sum_{k=0}^{m}\left(\begin{array}{c}
m \\
k
\end{array}\right) u(y)^{k} u(x)^{m-k} \\
& =\sum_{k=0}^{n} a_{k}(x) \sum_{y \in \Lambda} w(x, y) u(y)^{k} \\
& =\sum_{k=0}^{n} a_{k}(x)\left(G * u^{k}\right)(x)
\end{aligned}
$$


with $a_{k}(x)$ a polynomial depending on $u(x)$. The convolution can be computed in a fast way way using the fast Fourier transform. Note that in [7] not the sign function, but a continuous approximation of it, namely a slope function was approximated. Also for this function the Chebyhsev approximation will not stay within $[-1,1]$.

\section{B Remarks on the Continuous Case}

Let $\Omega$ the domain of the image which is considered as bounded with Lipschitz boundary. We define the average local contrast measure $u \in L^{2}(\Omega)$ as

$$
\mathrm{C}(u)=\int_{\Omega^{2}} w(x, y)|u(x)-u(y)| d x d y
$$

with $w(x, y) \in L^{2}\left(\Omega^{2}\right)$ a symmetric, non negative kernel such that

$$
\int_{\Omega} w(x, y) d x=1, \forall y \in \Omega .
$$

A typical kernel is

$$
w(x, y)=\frac{1}{\sigma \sqrt{2 \pi}} \mathrm{e}^{-\frac{\|x-y\|_{2}^{2}}{2 \sigma^{2}}}, \quad \sigma>0 .
$$

In order to enhance images, we propose a variational model for an image $u_{0}$ : $\Omega \rightarrow[0,1]:$

$$
\min _{u \in L^{2}(\Omega)}\left\|u-u_{0}\right\|_{L^{2}(\Omega)}^{2}+\lambda\left(\mathrm{C}(u)-c \mathrm{C}\left(u_{0}\right)\right)^{2}+\iota_{[0,1]}(u),
$$

where $\lambda>0$ manages the trade-off between the fidelity-data term and the contrast term. The functional (39) is positive and coercive in $L^{2}(\Omega)$. We show the existence of a minimizer of a regularized version of the functional (39) which was inspired from [7]. We set

$$
\begin{aligned}
F(u)+\lambda H(u):=\left\|u-u_{0}\right\|_{L^{2}}^{2} & \\
& +\lambda\left(\int_{\Omega^{2}} w(x, y)|u(x)-u(y)| d x d y-a\right)^{2},
\end{aligned}
$$

with a constant $a=c \mathrm{C}\left(u_{0}\right)$, and consider the regularized version

$$
F(u)+\lambda H(G * u),
$$

with a smoothing Gaussian kernel $G$. The following proposition appears to be useful.

Proposition 2. The functional $H(u)$ is continuous on its domain in $L^{2}(\Omega)$. 
Proof. We prove the continuity of $\mathrm{C}(u)$. Let us consider $\left\{u_{n}\right\}_{n}$ such that $u_{n} \rightarrow u$ in $L^{2}(\Omega)$ as $n \rightarrow \infty$. Then we obtain

$$
\begin{aligned}
\mathrm{C}\left(u_{n}\right)-\mathrm{C}(u) & =\int_{\Omega^{2}} w(x, y)\left|u_{n}(x)-u_{n}(y)\right| d x d y \\
& -\int_{\Omega^{2}} w(x, y)|u(x)-u(y)| d x d y \\
& =\int_{\Omega^{2}} w(x, y) \mid u_{n}(x)-u(x)-u(y) \\
& +u_{n}(y)+u(x)-u(y) \mid d x d y \\
& -\int_{\Omega^{2}} w(x, y)|u(x)-u(y)| d x d y
\end{aligned}
$$

Using the triangular inequality we conclude

$$
\begin{aligned}
& \left|\mathrm{C}\left(u_{n}\right)-\mathrm{C}(u)\right| \\
& \leq \int_{\Omega^{2}} w(x, y)\left(\left|u_{n}(x)-u(x)\right|+\left|u_{n}(y)-u(y)\right|\right) d x d y \\
& \leq 2 \int_{\Omega}\left|u_{n}(x)-u(x)\right| d x .
\end{aligned}
$$

Since we have convergence of $\left\{u_{n}\right\}_{n}$ in $L^{2}(\Omega)$ this implies also convergence in $L^{1}(\Omega)$. Hence $H(u)=(\mathrm{C}(u)-a)^{2}$ is continuous in $L^{2}(\Omega)$ on its domain.

Theorem 3. The functional $F(u)+\lambda H(G * u)$ admits a minimizer in $L^{2}(\Omega)$.

Proof. The functional $F(u)+\lambda H(G * u)$ is bounded from below. Let $\left\{u_{n}\right\}_{n}$ be a minimizing sequence, i.e.,

$$
F\left(u_{n}\right)+\lambda H\left(G * u_{n}\right) \underset{n \rightarrow+\infty}{\rightarrow} \inf _{v} F(v)+\lambda H(G * v) .
$$

Since $F\left(u_{n}\right)+\lambda H\left(G * u_{n}\right) \leq M$ for all $n \in \mathbb{N}$, we have $F\left(u_{n}\right) \leq M$, and it follows that $\left\|u_{n}\right\|_{L^{2}} \leq M$. Thus, up to a subsequence, there exists $\tilde{u}$ such that $u_{n} \rightarrow \tilde{u}$ in $L^{2}(\Omega)$ as $n \rightarrow+\infty$. Since $F$ is convex, we have that, (see, e.g., [9]):

$$
F(\tilde{u}) \leq \liminf _{n} F\left(u_{n}\right) .
$$

Since $u \mapsto G * u$ is a Hilbert-Schmidt operator, it is a compact operator in $L^{2}(\Omega)$. Thus, up to a subsequence, $G * u_{n} \rightarrow G * \tilde{u}$ as $n \rightarrow+\infty$ with strong convergence in $L^{2}(\Omega)$ (see, e.g., [9]). By Proposition 2 we get

$$
\lambda H\left(G * u_{n}\right) \underset{n \rightarrow+\infty}{\rightarrow} \lambda H(G * \tilde{u}) .
$$

Finally we obtain

$$
F(\tilde{u})+\lambda H(G * \tilde{u}) \leq \liminf _{n} F\left(u_{n}\right)+\lambda H\left(G * u_{n}\right) .
$$

Thus $\tilde{u}$ is a minimizer of $F(u)+\lambda H(G * u)$. 


\section{References}

[1] Abdullah-Al-Wadud, M., Kabir, M.H., Dewan, M., Chae, O.: A dynamic histogram equalization for image contrast enhancement. IEEE Transactions on Consumer Electronics 53(2), 593-600 (2007)

[2] Adelson, E.H.: Checkershadow illusion. Available at http://persci.mit.edu/gallery/checkershadow 2(1) (1995)

[3] Arici, T., Dikbas, S., Altunbasak, Y.: A histogram modification framework and its application for image contrast enhancement. IEEE Transactions on Image Processing 18(9), 1921-1935 (2009)

[4] Attouch, H., Bolte, J., Svaiter, B.F.: Convergence of descent methods for semi-algebraic and tame problems: proximal algorithms, forward-backward splitting, and regularized gauss-seidel methods. Mathematical Programming 137(1-2), 91-129 (2013)

[5] Aujol, J.F., Gilboa, G., Papadakis, N.: Fundamentals of non-local total variation spectral theory. Scale Space and Variational Methods in Computer Vision pp. 66-77 (2015)

[6] Bertalmío, M., Caselles, V., Provenzi, E.: Issues about retinex theory and contrast enhancement. International Journal of Computer Vision 83(1), 101-119 (2009)

[7] Bertalmío, M., Caselles, V., Provenzi, E., Rizzi, A.: Perceptual color correction through variational techniques. IEEE Transactions on Image Processing 16(4), 1058-1072 (2007)

[8] Boccignone, G., Picariello, A.: Multiscale contrast enhancement of medical images. In: IEEE International Conference on Acoustics, Speech, and Signal Processing, vol. 4, pp. 2789-2792 (1997)

[9] Brezis, H.: Analyse fonctionnelle. collection mathématiques appliquées pour la maltrise. (1983)

[10] Celik, T.: Two-dimensional histogram equalization and contrast enhancement. Pattern Recognition 45(10), 3810-3824 (2012)

[11] Celik, T., Tjahjadi, T.: Contextual and variational contrast enhancement. IEEE Transactions on Image Processing 20(12), 3431-3441 (2011)

[12] Celik, T., Tjahjadi, T.: Automatic image equalization and contrast enhancement using gaussian mixture modeling. IEEE Transactions on Image Processing 21(1), 145-156 (2012)

[13] Chambolle, A., Pock, T.: A first-order primal-dual algorithm for convex problems with applications to imaging. Journal of Mathematical Imaging and Vision 40(1), 120-145 (2011)

[14] Chambolle, A., Pock, T.: On the ergodic convergence rates of a first-order primal-dual algorithm. In: preprint (2014). URL http://www.optimization-online.org/DB_FILE/2014/09/4532.pdf 
[15] Chan, R., Nikolova, M., Wen, Y.W.: A variational approach for exact histogram specification. In: Scale Space and Variational Methods in Computer Vision, pp. 86-97 (2012)

[16] Chen, S.D., Ramli, A.R.: Contrast enhancement using recursive meanseparate histogram equalization for scalable brightness preservation. IEEE Transactions on Consumer Electronics 49(4), 1301-1309 (2003)

[17] Coltuc, D., Bolon, P., Chassery, J.M.: Exact histogram specification. IEEE Transactions on Image Processing 15(6), 1143-1152 (2006)

[18] Ferradans, S., Palma-Amestoy, R., Provenzi, E.: An algorithmic analysis of variational models for perceptual local contrast enhancement. Image Processing On Line 5, 219-233 (2015)

[19] Fitschen, J.H., Nikolova, M., Pierre, F., Steidl, G.: A variational model for color assignment. In: Scale Space and Variational Methods in Computer Vision, pp. 437-448 (2015)

[20] Gatta, C., Rizzi, A., Marini, D.: Ace: An automatic color equalization algorithm. In: Conference on Colour in Graphics, Imaging, and Vision, vol. 1, pp. 316-320 (2002)

[21] Getreuer, P.: Automatic Color Enhancement (ACE) and its Fast Implementation. Image Processing On Line 2, 266-277 (2012). DOI 10.5201/ipol.2012.g-ace

[22] Gilboa, G., Osher, S.: Nonlocal operators with applications to image processing. Multiscale Modeling \& Simulation 7(3), 1005-1028 (2008)

[23] Gonzalez, R.C., Wintz, P.: Digital Image Processing, second edn. AddisonWesley, Reading (2007)

[24] Gonzalez, R.C., Woods, R.E.: Digital image processing 3rd edition (2007)

[25] Hummel, R.: Image enhancement by histogram transformation. Computer graphics and image processing 6(2), 184-195 (1977)

[26] Häuser, S., Nikolova, M., Steidl, G.: Hue and range preserving rgb image enhancement (rgb-hp-enhance). Preprint (2015). Documentation for Matlab toolbox

[27] Jobson, D.J., Rahman, Z.u., Woodell, G.: Properties and performance of a center/surround retinex. IEEE Transactions on Image Processing 6(3), 451-462 (1997)

[28] Kaur, M., Kaur, J., Kaur, J.: Survey of contrast enhancement techniques based on histogram equalization. International Journal of Advanced Computer Science and Applications 2(7), 137-141 (2011)

[29] Kim, Y.T.: Contrast enhancement using brightness preserving bi-histogram equalization. IEEE Transactions on Consumer Electronics 43(1), 1-8 (1997) 
[30] Laine, A., Fan, J., Yang, W.: Wavelets for contrast enhancement of digital mammography. IEEE Engineering in Medicine and Biology Magazine 14(5), 536-550 (1995)

[31] Land, E.H., McCann, J.: Lightness and retinex theory. Journal of the Optical Society of America 61(1), 1-11 (1971)

[32] Łojasiewicz, S.: Sur le probleme de la division. Studia Mathematica XVIII, 87-136 (1961)

[33] Maini, R., Aggarwal, H.: A comprehensive review of image enhancement techniques. Journal of Computing 2(3), 919-940 (2010)

[34] Mignotte, M.: An energy-based model for the image edge-histogram specification problem. IEEE Transactions on Image Processing 21(1), 379-386 (2012)

[35] Nikolova, M.: A fast algorithm for exact histogram specification. simple extension to colour images. In: Scale Space and Variational Methods in Computer Vision, pp. 174-185 (2013)

[36] Nikolova, M., Steidl, G.: Fast hue and range preserving histogram specification: Theory and new algorithms for color image enhancement. IEEE Transactions on Image Processing 23(9), 4087-4100 (2014)

[37] Nikolova, M., Steidl, G.: Fast ordering algorithm for exact histogram specification. IEEE Transactions on Image Processing 23(12), 5274-5283 (2014)

[38] Nikolova, M., Wen, Y.W., Chan, R.: Exact histogram specification for digital images using a variational approach. Journal of Mathematical Imaging and Vision 46(3), 309-325 (2013)

[39] Osher, S., Rudin, L.I.: Feature-oriented image enhancement using shock filters. SIAM Journal on Numerical Analysis 27(4), 919-940 (1990)

[40] Palma-Amestoy, R., Provenzi, E., Bertalmío, M., Caselles, V.: A perceptually inspired variational framework for color enhancement. IEEE Transactions on Pattern Analysis and Machine Intelligence 31(3), 458-474 (2009)

[41] Papadakis, N., Provenzi, E., Caselles, V.: A variational model for histogram transfer of color images. IEEE Transactions on Image Processing 20(6), 1682-1695 (2011)

[42] Perona, P., Malik, J.: Scale-space and edge detection using anisotropic diffusion. IEEE Transactions on Pattern Analysis and Machine Intelligence 12(7), 629-639 (1990)

[43] Piella, G.: Image fusion for enhanced visualization: A variational approach. International Journal of Computer Vision 83(1), 1-11 (2009)

[44] Pierre, F., Aujol, J.F., Bugeau, A., Ta, V.T.: Luminance-hue specification in the rgb space. In: Scale Space and Variational Methods in Computer Vision, pp. 413-424 (2015) 
[45] Pierre, F., Migerditichan, P.: Débrumage variationnel. In: XXVème colloque GRETSI, pp. 1-4 (2015)

[46] Pock, T., Chambolle, A., Cremers, D., Bischof, H.: A convex relaxation approach for computing minimal partitions. In: IEEE Conf. Computer Vision and Pattern Recognition, pp. 810-817 (2009)

[47] Provenzi, E., Caselles, V.: A wavelet perspective on variational perceptually-inspired color enhancement. International Journal of Computer Vision 106(2), 153-171 (2014)

[48] Rizzi, A., Gatta, C., Marini, D.: A new algorithm for unsupervised global and local color correction. Pattern Recognition Letters 24(11), 1663-1677 (2003)

[49] Rizzi, A., Gatta, C., Marini, D.: From retinex to automatic color equalization: issues in developing a new algorithm for unsupervised color equalization. Journal of Electronic Imaging 13(1), 75-84 (2004)

[50] Rudin, L.I., Osher, S., Fatemi, E.: Nonlinear total variation based noise removal algorithms. Physica D: Nonlinear Phenomena 60(1), 259-268 (1992)

[51] Sapiro, G., Caselles, V.: Histogram modification via differential equations. Journal of Differential Equations 135(2), 238-268 (1997)

[52] Sim, K., Tso, C., Tan, Y.: Recursive sub-image histogram equalization applied to gray scale images. Pattern Recognition Letters 28(10), 1209 1221 (2007)

[53] Sugimura, D., Mikami, T., Yamashita, H., Hamamoto, T.: Enhancing color images of extremely low light scenes based on $\mathrm{rgb} / \mathrm{nir}$ images acquisition with different exposure times. IEEE Transactions on Image Processing (2015)

[54] Sun, C.C., Ruan, S.J., Shie, M.C., Pai, T.W.: Dynamic contrast enhancement based on histogram specification. IEEE Transactions on Consumer Electronics 51(4), 1300-1305 (2005)

[55] Wan, Y., Shi, D.: Joint exact histogram specification and image enhancement through the wavelet transform. IEEE Transactions on Image Processing 16(9), 2245-2250 (2007)

[56] Wang, C., Ye, Z.: Brightness preserving histogram equalization with maximum entropy: a variational perspective. IEEE Transactions on Consumer Electronics 51(4), 1326-1334 (2005)

[57] Zhu, M., Chan, T.: An efficient primal-dual hybrid gradient algorithm for total variation image restoration. Tech. rep., UCLA, Center for Applied Math. (2008) 